\title{
Research Article \\ Effects of Separation Strategy on Deployment of Multitethered Chain-Type Satellite System
}

\author{
Jinxiu Zhang and Zhigang Zhang \\ Research Center of Satellite Technology, Harbin Institute of Technology, Harbin 150080, China \\ Correspondence should be addressed to Zhigang Zhang; zhzhghit@126.com
}

Received 10 April 2016; Accepted 19 September 2016

Academic Editor: Yuri Vladimirovich Mikhlin

Copyright ( 92016 J. Zhang and Z. Zhang. This is an open access article distributed under the Creative Commons Attribution License, which permits unrestricted use, distribution, and reproduction in any medium, provided the original work is properly cited.

\begin{abstract}
This paper investigates the effects of separation strategy and parameters related to deployment on the dynamic behavior of multitethered chain-type satellite system. The system, including several satellites connected by tethers which are considered as massless and straight, is modeled as an extension of a two-body dumbbell tethered system. The dynamic equations of system in absence of perturbations and external disturbances are derived using Newtonian Method. To observe the effect of deployment rate on the motion of system, a parametric analysis of the deployment of a three-body tethered system with different deployment rates is carried out. Moreover, a four-body tethered system is used to investigate the effect of separation strategies on the dynamic behavior of system during the deployment phase. The numerical results suggest that the system with simultaneous separation costs less time to complete the deployment. If the ratio of deployment rates is in consistence with that of their desired lengths, the tethers deployed simultaneously would have a synchronous motion. It is also observed that the system employing separation bolt has a better performance than the system separated by spring mechanism since the larger separation velocity which is not along local vertical may cause a rotation.
\end{abstract}

\section{Introduction}

Tethered satellite system (TSS) is an interesting concept, made up of several satellites linked by the means of tethers, and has received increasing attention due to its potential for many important space applications [1], such as investigation of planetary atmosphere, micro-gravity experiments, energy transmission, orbit maneuvers, orbit maintenances, and space formations as platform for interferometry. Recently multitethered satellite system has attracted researchers' attention and has been conceived for several space missions. The key characteristic making this system appealing is the lightness of tethers; thus the system could keep together or adjust its configuration with little fuel consumption, which would be beneficial for many space missions, especially for space remote interferometry. Moreover, the multitethered chain-type satellite system could provide the space remote interferometry with single-direction baselines and the objective of this research is to study the affecting factors of its deployment to obtain single-direction baselines.
A successful deployment of TSS is the prerequisite for space missions. Much attention has been paid to deployment of TSS and many space experiments were performed in order to investigate the dynamics of TSS and validate the control methods $[2,3]$. Different kinds of methods were put forward to complete the deployment of TSS. A passive deployment procedure was proposed to bring a payload into a permanent locally vertical position [4]. Misra and Modi investigated the deployment and retrieval of TTS basing on a general dynamical model and figured out that the in-plane motion could be controlled by the deviation between the deployment rate of the undeformed tether and a commanded rate obtained by the state vector combining linearly [5]. An optimal control strategy of deployment of a tethered satellite system was developed and compared with free deployment and deployment method of Kissel's law [6]. Williams studied the optimal deployment and retrieval trajectories via numerical method under various optimality criteria and found the criteria incorporating the minimization of system accelerations is more appropriate [7]. Free and nonfree deployment of a space 
tether-net system were carried out numerically to study the dynamic behavior of the system during deployment [8]. Sun and Zhu developed a new fractional-order tension control law to deploy a space tether fast and stable [9]. Zakrzhevskii proposed a tether length control law based on the changeof-angular-momentum theorem to deploy a tethered space system along the local vertical [10]. Fruitful results of the dynamic and control of TSS have been obtained from the theoretical analyses, numerical and experimental validations. However, the interest of most researches was focused on a two-body tethered system, and less attention was given to the multitethered satellite system.

The nonlinear dynamics of multitethered satellite system is complicated. Most research about the multitethered satellite system was concentrated on the dynamics analysis of station-keeping phase. A general three-dimensional equation of motion of tethered $N$-body systems was derived and the dynamic behavior during the station-keeping phase was investigated via numerical simulations [11]. The equilibrium configurations of a four-body tethered system and a tethered three-body system were obtained by Corrêa and Gómez [12] and $\mathrm{Xu}$ et al. [13], respectively. The dynamic behavior of a multitethered satellite formation, given the tether mass, gravity gradient force, and tether tension, was analyzed by Avanzini and Fedi [14] and the effects of eccentricity on the dynamics of system were examined by them as well [15]. The equilibrium conditions of a triple-mass triple-tethered satellite system moving on a low Earth orbit were found and the stability is investigated [16]. Alary et al. focused their attention on the dynamics of a multitethered pyramidal formation and proposed a control strategy in order to stabilize the system [17]. The dynamic behavior of a three-body tethered satellite system was parametrically analyzed under several groups of initial satellite attitudes by Jung et al. [18]. To the author's knowledge, most attention was focused on the dynamics model and stability around the nominal position, that is, the equilibrium situation, and control methods, which intend to stabilize the system to the equilibrium position. However, there has been little research on the deployment strategy of multitethered satellite, which plays an important role in the tethered missions.

This paper intends to study the effects of separation strategies on the dynamic behavior of multitethered chaintype satellite system. The system consists of several satellites and tethers attached to them and could be divided into several elements, which only has two satellites and one tether. As a consequence, the deployment methods suitable for two-body system could be applied for the deployment of one element directly. However, the effects of deployment of one element on the dynamics of the system, which is an actual problem for the multitethered chain-type satellite system, is not investigated yet. Hence, the dynamic behavior of the system with different separation strategies will be studied in this paper.

To deploy the system along vertical or horizontal direction and obtain single-direction baselines, the dynamic equations of a multitethered satellite system are derived by means of Newtonian Method as the start in Section 2. Subsequently, the separation strategies and control method of deployment are given in Sections 3 and 4, respectively. Afterwards, the

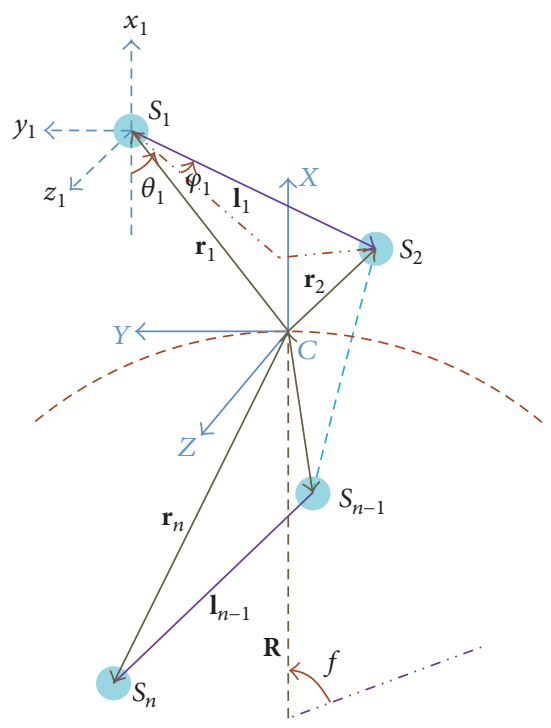

Figure 1: Dynamic model of a multitethered satellite system.

effects of the separation strategy and deployment rate on the dynamic behavior during deployment are parametrically analyzed in Section 5.

\section{Dynamic Equations of Multitethered Chain-Type Satellite System}

The multitethered satellite system is composed of several satellites connected by the means of tethers. Since the dimensional size of satellites is much less than the length of tether, the satellites are assumed as point masses, which means that the attitude of satellites is neglected in this paper. Meanwhile, the mass of tethers is far less than that of satellites. For simplicity and focusing attention on the effects of separation strategy on the deployment, the tethers are considered as massless and straight. Additionally, some sensible assumptions are made for the same purpose: (1) the center of mass of the system moves on a Keplerian orbit, (2) the perturbations such as atmospheric drag and Earth oblateness, are negligible, and (3) the eccentricity of the orbit is small and would not induce the system into chaos motion [19].

The dynamic behavior of the multitethered satellite system is depicted with respect to the orbital frame $C X Y Z$ with its origin at the center of mass of the system, as shown in Figure 1. The $C X$ axis points outwards along the radial direction, $C Z$ axis is perpendicular to the orbital plane, coinciding with the direction of orbit angular velocity, and the $C Y$ axis completes a right-handed orthogonal triad.

Since multitethered chain-type satellite system consists of $N$ satellites connected by $N-1$ tethers in a chain configuration, the dynamics of the model could be described by $3 N$ generalized coordinates. Meanwhile, the center of mass of the system moves on a Keplerian orbit, which could be ascertained by the orbital dynamics; then the dynamic of system could be represented by $3(N-1)$ generalized coordinates. In order that the control method of deployment could be 
conveyed conveniently with the generalized coordinates, the descriptive parameters of tether, that is, $l_{k}$ representing the length of $k$ th tether, $\theta_{k}$ depicting the librational angle from local vertical in orbital plane, and $\varphi_{k}$ meaning the out-ofplane angle, are chosen to develop the dynamics model of the system.

The equations of motion for the system are formulated via a straightforward Newtonian approach. For $l_{k}$ connecting satellites $S_{k}$ and $S_{k+1}$, the vector form of it could be described as

$$
\mathbf{l}_{k}=\mathbf{R}_{k+1}-\mathbf{R}_{k}
$$

where $\mathbf{R}_{k}$ denotes the position vector of $S_{k}$ with respect to Earth's center, and it could be written as

$$
\mathbf{R}_{k}=\mathbf{R}+\mathbf{r}_{k}
$$

where $\mathbf{R}, \mathbf{r}_{k}$ represent the vectors from Earth's center to the centroid of the system and the centroid of system to satellite $S_{k}$, respectively.

Since the origin of orbital frame is at the center of mass of system, then according to the material center theorem there has been

$$
\sum_{k=1}^{N} m_{k} \mathbf{r}_{k}=0
$$

Moreover, the relation between position vectors of adjacent satellites with respect to centroid of system could be written as

$$
\mathbf{r}_{k+1}=\mathbf{r}_{k}+\mathbf{l}_{k}
$$

Substituting (4) into (3) and performing some algebraic operations, the position vector of $k$ th satellite is represented as

$$
\mathbf{r}_{k}=\frac{1}{m}\left(\sum_{i=1}^{k-1} A_{1}^{i} \mathbf{l}_{i}-\sum_{i=k}^{N} A_{i+1}^{N} \mathbf{l}_{i}\right),
$$

where $m$ is the total mass of the system; that is, $m=$ $\sum_{k=1}^{N} m_{k}, A_{i}^{j}=\sum_{k=i}^{j} m_{k}$.

Project the $k$ th tether to the axes of the orbital frame CXYZ:

$$
\mathbf{l}_{k}=\left[\begin{array}{c}
-l_{k} \cos \varphi_{k} \cos \theta_{k} \\
-l_{k} \cos \varphi_{k} \sin \theta_{k} \\
l_{k} \sin \varphi_{k}
\end{array}\right] .
$$

The angular velocity of the orbital frame could be written as

$$
\boldsymbol{\omega}=\left[\begin{array}{l}
0 \\
0 \\
\dot{f}
\end{array}\right] \text {, }
$$

where $f$ means the true anomaly of the center of mass of the system.
Hence, the two-order derivative of $\mathbf{l}_{k}$ with respect to time could be calculated:

$$
\ddot{\mathbf{l}}_{k}=\left[\begin{array}{l}
\ddot{l}_{k x} \\
\ddot{l}_{k y} \\
\ddot{l}_{k z}
\end{array}\right]
$$

where

$$
\begin{aligned}
\ddot{l}_{k x}= & -\ddot{l}_{k} \cos \theta_{k} \cos \varphi_{k}+l_{k} \ddot{\varphi}_{k} \cos \theta_{k} \sin \varphi_{k} \\
& +l_{k} \ddot{\theta}_{k} \sin \theta_{k} \cos \varphi_{k}+2 \dot{l}_{k} \dot{\varphi}_{k} \cos \theta_{k} \sin \varphi_{k} \\
& +2 \dot{l}_{k}\left(\dot{\theta}_{k}+\omega\right) \sin \theta_{k} \cos \varphi_{k} \\
& +l_{k} \dot{\omega} \sin \theta_{k} \cos \varphi_{k} \\
& +l_{k}\left[\dot{\varphi}_{k}^{2}+\left(\dot{\theta}_{k}+\omega\right)^{2}\right] \cos \theta_{k} \cos \varphi_{k} \\
& -2 l_{k} \dot{\varphi}_{k}\left(\dot{\theta}_{k}+\omega\right) \sin \theta_{k} \sin \varphi_{k}, \\
\ddot{l}_{k y}= & -\ddot{l}_{k} \sin \theta_{k} \cos \varphi_{k}+l_{k} \ddot{\varphi}_{k} \sin \theta_{k} \sin \varphi_{k} \\
& -l_{k} \ddot{\theta}_{k} \cos \theta_{k} \cos \varphi_{k}+2 \dot{l}_{k} \dot{\varphi}_{k} \sin \theta_{k} \sin \varphi_{k} \\
& -2 \dot{l}_{k}\left(\dot{\theta}_{k}+\omega\right) \cos \theta_{k} \cos \varphi_{k} \\
& -l_{k} \dot{\omega} \cos \theta_{k} \cos \varphi_{k} \\
& +l_{k}\left[\dot{\varphi}_{k}^{2}+\left(\dot{\theta}_{k}+\omega\right)^{2}\right] \sin \theta_{k} \cos \varphi_{k} \\
& +2 l_{k} \dot{\varphi}_{k}\left(\dot{\theta}_{k}+\omega\right) \cos \theta_{k} \sin \varphi_{k}, \\
\ddot{l}_{k z}= & \ddot{l}_{k} \sin \varphi_{k}+l_{k} \ddot{\varphi}_{k} \cos \varphi_{k}-l_{k} \dot{\varphi}_{k}^{2} \sin \varphi_{k} \\
& \cos \varphi_{k} .
\end{aligned}
$$

Meanwhile, with (1) we could obtain

$$
\ddot{\mathbf{l}}_{k}=\ddot{\mathbf{R}}_{k+1}-\ddot{\mathbf{R}}_{k} \text {. }
$$

The acceleration of satellite $S_{k}$ is determined by the forces acting on it, as shown in Figure 2.

According to Newton's Second Law, we have

$$
m_{k} \ddot{\mathbf{R}}_{k}=\mathbf{G}_{k}+\mathbf{T}_{k}-\mathbf{T}_{k-1}
$$

where $\mathbf{G}_{k}$ is the gravitational force and $\mathbf{T}_{k}$ is the tether tension due to the stretch of $k$ th tether.

The gravitational force $\mathbf{G}_{k}$ could be obtained by

$$
\mathbf{G}_{k}=-\frac{\mu m_{k}}{\left|\mathbf{R}_{k}\right|^{3}} \mathbf{R}_{k} .
$$

Substituting (2) and expanding (12) into a binomial series and then neglecting the terms of fourth and higher orders, which is negligible since $\mathbf{r}_{k} \ll \mathbf{R}$, we could acquire

$$
\mathbf{G}_{k} \approx-\mu m_{k} R^{-3}\left(\mathbf{R}+\mathbf{r}_{k}\right)\left[1-3 \frac{\mathbf{R} \cdot \mathbf{r}_{k}}{R^{2}}\right] \text {. }
$$




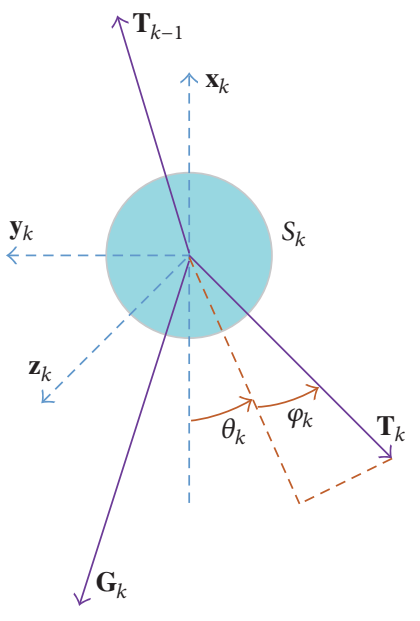

FIGURE 2: The forces acting on satellite $S_{k}$.

Then (11) could be rewritten as

$$
\ddot{\mathbf{R}}_{k} \approx-\mu R^{-3}\left(\mathbf{R}+\mathbf{r}_{k}\right)\left[1-3 \frac{\mathbf{R} \cdot \mathbf{r}_{k}}{R^{2}}\right]+\frac{\mathbf{T}_{k}-\mathbf{T}_{k-1}}{m_{k}}
$$

The dynamic equations of satellite $S_{k+1}$ are similar with $S_{k}$, and by replacing $\ddot{\mathbf{R}}_{k}$ and $\ddot{\mathbf{R}}_{k+1}$ in (10) with (14), one obtains another representation of $\ddot{\mathbf{l}}_{k}$ :

$$
\begin{aligned}
\ddot{\mathbf{l}}_{k} \approx & -\mu R^{-3}\left[\mathbf{l}_{k}-\frac{3}{R^{2}}\left(\mathbf{R} \cdot \mathbf{l}_{k}\right) \mathbf{R}\right]+\frac{\mathbf{T}_{k+1}-\mathbf{T}_{k}}{m_{k+1}} \\
& -\frac{\mathbf{T}_{k}-\mathbf{T}_{k-1}}{m_{k}} .
\end{aligned}
$$

Obviously, the two expressions of $\ddot{\mathbf{l}}_{k}$ are equivalent. Hence, the right parts of both equations are equal and by some algebraic manipulations, we can obtain the dynamic equations for $k$ th tether:

$$
\mathbf{M}_{k} \ddot{\mathbf{x}}_{k}=F_{k}\left(\mathbf{x}_{k}, \dot{\mathbf{x}}_{k}, \boldsymbol{\omega}, \dot{\boldsymbol{\omega}}\right)
$$

where $\mathbf{x}_{k}=\left[\begin{array}{lll}l_{k} & \theta_{k} & \varphi_{k}\end{array}\right]^{T}$ is the combination of generalized coordinates corresponding to $k$ th tether and $\mathbf{M}_{k}$ is a matrix dependent on $\mathbf{x}_{k}$ :

$$
\begin{aligned}
& \mathbf{M}_{k} \\
& =\left[\begin{array}{ccc}
-\cos \theta_{k} \cos \varphi_{k} & l_{k} \sin \theta_{k} \cos \varphi_{k} & l_{k} \cos \theta_{k} \sin \varphi_{k} \\
-\sin \theta_{k} \cos \varphi_{k} & -l_{k} \cos \theta_{k} \cos \varphi_{k} & l_{k} \sin \theta_{k} \sin \varphi_{k} \\
\sin \varphi_{k} & 0 & l_{k} \cos \varphi_{k}
\end{array}\right] .
\end{aligned}
$$

The right part $F_{k}(\cdot)$ is a function of generalized coordinates and generalized speeds of them, as well as the orbit angular velocity and its derivative with respect to time. The components projecting on $C X Y Z$, namely, $F_{k x}, F_{k y}$, and $F_{k z}$, have the form as

$$
\begin{aligned}
& F_{k x}=-2 \mu R^{-3} l_{k} \cos \theta_{k} \cos \varphi_{k}-2 \dot{l}_{k} \dot{\varphi}_{k} \cos \theta_{k} \sin \varphi_{k} \\
& -2 \dot{l}_{k}\left(\dot{\theta}_{k}+\omega\right) \sin \theta_{k} \cos \varphi_{k} \\
& -l_{k} \dot{\omega} \sin \theta_{k} \cos \varphi_{k} \\
& -l_{k}\left[\dot{\varphi}_{k}^{2}+\left(\dot{\theta}_{k}+\omega\right)^{2}\right] \cos \theta_{k} \cos \varphi_{k} \\
& +2 l_{k} \dot{\varphi}_{k}\left(\dot{\theta}_{k}+\omega\right) \sin \theta_{k} \sin \varphi_{k} \\
& -\frac{\mathbf{T}_{k+1} \cos \theta_{k+1} \cos \varphi_{k+1}-\mathbf{T}_{k} \cos \theta_{k} \cos \varphi_{k}}{m_{k+1}} \\
& +\frac{\mathbf{T}_{k} \cos \theta_{k} \cos \varphi_{k}-\mathbf{T}_{k-1} \cos \theta_{k-1} \cos \varphi_{k-1}}{m_{k}}, \\
& F_{k y}=\mu R^{-3} l_{k} \sin \theta_{k} \cos \varphi_{k}-2 \dot{l}_{k} \dot{\varphi}_{k} \sin \theta_{k} \sin \varphi_{k} \\
& +2 \dot{l}_{k}\left(\dot{\theta}_{k}+\omega\right) \cos \theta_{k} \cos \varphi_{k} \\
& +l_{k} \dot{\omega} \cos \theta_{k} \cos \varphi_{k} \\
& -l_{k}\left[\dot{\varphi}_{k}^{2}+\left(\dot{\theta}_{k}+\omega\right)^{2}\right] \sin \theta_{k} \cos \varphi_{k} \\
& -2 l_{k} \dot{\varphi}_{k}\left(\dot{\theta}_{k}+\omega\right) \cos \theta_{k} \sin \varphi_{k} \\
& -\frac{\mathbf{T}_{k+1} \sin \theta_{k+1} \cos \varphi_{k+1}-\mathbf{T}_{k} \sin \theta_{k} \cos \varphi_{k}}{m_{k+1}} \\
& +\frac{\mathbf{T}_{k} \sin \theta_{k} \cos \varphi_{k}-\mathbf{T}_{k-1} \sin \theta_{k-1} \cos \varphi_{k-1}}{m_{k}}, \\
& F_{k z}=-\mu R^{-3} l_{k} \sin \varphi_{k}-2 \dot{l}_{k} \dot{\varphi}_{k} \cos \varphi_{k}+l_{k} \dot{\varphi}_{k}^{2} \sin \varphi_{k} \\
& +\frac{\mathbf{T}_{k+1} \sin \varphi_{k+1}-\mathbf{T}_{k} \sin \varphi_{k}}{m_{k+1}} \\
& -\frac{\mathbf{T}_{k} \sin \varphi_{k}-\mathbf{T}_{k-1} \sin \varphi_{k-1}}{m_{k}} .
\end{aligned}
$$

It is worthy to note that the dynamic equations above are the complete form for one tether. For the end tether $(k=$ $1, N)$, the force $\mathbf{T}_{k-1}$ or $\mathbf{T}_{k+1}$ vanishes. The tether tension due to the stretch of tether could be obtained via Hooke's Law:

$$
\mathbf{T}_{k}=E A \frac{\delta l_{k}}{l_{k d}}=E A \frac{l_{k}-l_{k d}}{l_{k d}},
$$

where $E$ is the modulus of elasticity and $\delta l_{k}$ and $l_{k d}$ are the deformation and unstrained length of $k$ th tether, respectively.

The instantaneous radius of the center of mass of the system and the orbital angular velocity could be obtained via the orbital kinematics:

$$
\begin{aligned}
& R=\frac{a\left(1-e^{2}\right)}{1+e \cos f}, \\
& \omega=(1+e \cos f)^{2} \sqrt{\frac{\mu}{a^{3}\left(1-e^{2}\right)^{3}}},
\end{aligned}
$$




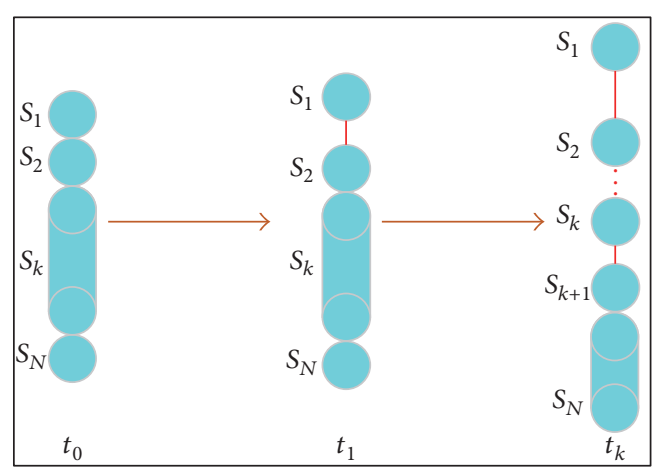

(a)

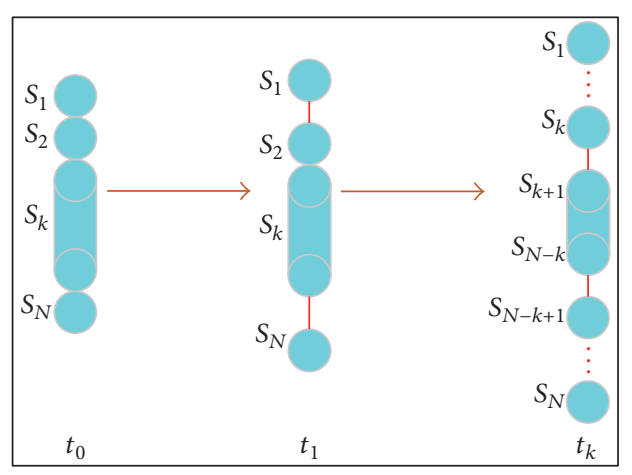

(b)

FIGURE 3: Two separation approaches of multitethered chain-type satellite system: (a) ejecting satellite outwards successively; (b) ejecting two satellites outwards simultaneously.

where $a$ is the semimajor axis of the orbital, $e$ is the eccentricity, and $\mu$ is the gravitational constant of Earth; here $\mu=3.986 \times 10^{14} \mathrm{~m}^{3} / \mathrm{s}^{2}$.

In addition, the angular acceleration of the orbit used in the dynamic equations could be got as

$$
\dot{\omega}=-2 \mu \frac{e \sin f(1+e \cos f)^{3}}{a^{3}\left(1-e^{2}\right)^{3}} .
$$

It should be noted that the deployment phase is shortterm and the tethers used in this paper are not long, such that the $J_{2}$ perturbations and atmospheric drag are excluded for the purpose of preliminary analysis of the effects of the separation sequences and deployment rate on the deployment. Additionally, the tensile elongation has been neglected during deployment since the variation of tether length is dominated by the deployment rate in this process. Once the tether length grows to the desired value, the deployment stops. Then the length of unstretched tether is fixed. If the tether length is bigger than the fixed value, the tether tension arises and could be approximately expressed as (19). Otherwise, the tether is slack and the tether tension does not exist.

\section{Separation Strategies of Multitethered Chain-Type Satellite System}

In contrast to the two-body system, which only requires one separation to initialize the deployment, the multitethered system needs several separations to deploy all the satellites. There exist two approaches of the separations: (1) the satellites are ejected one by one; for instance, the satellite $S_{k+2}$ is separated from $S_{k+1}$ only after the accomplishment of the deployment of the $k$ th tether; (2) all the satellites are separated with their neighbors at the same moment.

For the latter approach, if there exist some deviations from the prescribed separations it would lead to collisions between satellites due to the really small initial distance, which is the most undesirable situation for the space formation missions. Hence, if the simultaneous separation needs to execute, some modifications should be made to the second approach. To avoid the collision, only two satellites are ejected simultaneously and they should not be adjacent except for the first separation. The illustration of two approaches is seen in Figure 3.

Actually, the ejection usually is carried out via spring mechanism and offers the initial velocity of the deployment [20]. However, it is adverse for the adjacent tether which is fully deployed already. Another separation approach could be achieved by the means of separation bolt with low impact [21]. The impact on the deployed tether of this method decreases comparing to the former approach while the initial deployment rate decreases as well. As a result, the selection of the separation strategy has an important role in the deployment of the system and we intend to figure out the effects of the different separation approach on the dynamic behavior of deployment for a multitethered chain-type satellite system.

Both methods of separation are fulfilled by the internal force and the momentum conservation is effective before and after the separation. Assuming the separation between satellites $S_{k+1}$ and $S_{k}$, they move together with a velocity of $\mathbf{v}_{0}$ before the separation and have the velocities of $\mathbf{v}_{1}$ and $\mathbf{v}_{2}$, respectively, after the separation. For the ejection via spring mechanism, we obtain

$$
\begin{aligned}
m_{k+1} \mathbf{v}_{1}+m_{k} \mathbf{v}_{2} & =\left(m_{k}+m_{k+1}\right) \mathbf{v}_{0}, \\
\mathbf{v}_{1}-\mathbf{v}_{2} & =\dot{\mathbf{l}}_{k},
\end{aligned}
$$

where $\dot{\mathbf{l}}_{k}$ is the initial deployment velocity of the $k$ th tether, determined by the stored energy of springs and direction of the ejection.

Then the velocities after the ejection could be calculated:

$$
\begin{aligned}
& \mathbf{v}_{1}=\mathbf{v}_{0}+\frac{m_{k}}{m_{k}+m_{k+1}} \dot{\mathbf{l}}_{k}, \\
& \mathbf{v}_{2}=\mathbf{v}_{0}-\frac{m_{k+1}}{m_{k}+m_{k+1}} \dot{\mathbf{l}}_{k} .
\end{aligned}
$$

For the latter approach, the velocity of satellite $S_{k}$ decreases dramatically after separation and is almost near zero due to the tether tension of $(k-1)$ th tether. Meanwhile, 
the velocity of satellite $S_{k+1}$ remains unchanged. Then the initial deployment rate is obtained:

$$
\dot{\mathbf{l}}_{k}=\mathbf{v}_{0} \text {. }
$$

\section{Control Method of Deployment}

Tether deployment is one of the most important steps for the space tether missions. As mentioned before, there are many control laws of deployment proposed by the researchers. Here an effective and simple deployment method, which includes an exponential deployment phase, followed by a uniform velocity deployment and ending in an exponential manner again, is selected to focus attention on the study of the effects of separation strategy. According to [22], the deploy speed for the $k$ th tether is expressed as follows:

$$
\dot{l}_{k}= \begin{cases}c_{k} l_{k}, & L_{k i} \leq l_{k} \leq L_{k 1}, \\ c_{k} L_{k 1}, & L_{k 1} \leq l_{k} \leq L_{k 2}, \\ c_{k}\left(L_{k 1}+L_{k 2}-l_{k}\right), & L_{k 2} \leq l_{k} \leq L_{k f},\end{cases}
$$

where $L_{k 0}$ and $L_{k f}$ are the initial and desired length of $k$ th tether, $L_{k 1}$ and $L_{k 2}$ are the values where the deployment transitions from exponential rule to uniform instruction or vice versa, and $c_{k}$ is a positive constant selected with tryouts.

Fundamentally, the deployment of system could be implemented due to the gravitational gradient and centrifugal forces, both of which are determined by the distances of satellites with respect to the center of mass of system. However, at the beginning of the deployment the distance between satellites is very small and the gravitational gradient and centrifugal forces are trifling in comparison with the friction of the deployment mechanism, which is certainly adverse for the deployment. Moreover, the initial deploying velocity calculated by (25) is small as well and it would prolong the duration of the deployment. Hence, the deploy speed is modified and an extra velocity $c_{k} s_{k}$ is provided by the means of separation:

$$
\dot{l}_{k}= \begin{cases}c_{k}\left(l_{k}+s_{k}\right), & L_{k i} \leq l_{k} \leq L_{k 1}, \\ c_{k}\left(L_{k 1}+s_{k}\right), & L_{k 1} \leq l_{k} \leq L_{k 2}-s_{k}, \\ c_{k}\left(L_{k 1}+L_{k 2}-l_{k}\right), & L_{k 2}-s_{k} \leq l_{k} \leq L_{k f} .\end{cases}
$$

The deployment of a three-body tethered satellite system is carried out with the control method of (25), which is similar with methods described in $[18,22]$ and (26), respectively. The former method is denoted as the first method while the latter one is regarded as the second method. The relevant parameters used are as follows:

$$
\begin{aligned}
& m_{1}=m_{2}=m_{3}=200 \mathrm{~kg}, \\
& L_{10}=L_{20}=2 \mathrm{~m}, \\
& L_{1 f}=L_{2 f}=1000 \mathrm{~m},
\end{aligned}
$$

$$
\begin{aligned}
L_{11} & =L_{21}=250 \mathrm{~m}, \\
L_{12} & =L_{22}=752 \mathrm{~m}, \\
\theta_{10} & =\theta_{20}=0, \\
\dot{\theta}_{10} & =\dot{\theta}_{20}=0, \\
\varphi_{10} & =\varphi_{20}=0, \\
\dot{\varphi}_{10} & =\dot{\varphi}_{20}=0, \\
c_{1} & =c_{2}=4 \times 10^{-3} \mathrm{~s}^{-1}, \\
s_{1} & =s_{2}=50 \mathrm{~m} .
\end{aligned}
$$

The configurations of the system during the deployment are shown in Figure 4. Only the motion in orbital plane of satellites is given as the components along the normal direction maintain zero during the deployment. The satellite $S_{3}$ is deployed downwards along the local vertical and $S_{1}$ heads off to the opposite trend. As depicted in Figure 4(a), the system begins to spin around the centroid with the first method of deployment. On the contrary, the system turns into a pendulum motion when the deployment is completed in Figure 4(b). One reason for this may be that a larger initial velocity makes the distance enlarge much faster, thus larger restoring force, that is, the summation of gravitational and centrifugal forces, is produced and drives the system swing back towards local vertical. The second method is superior to the former one, which drives the system into a rotation.

\section{Numerical Simulation}

The complex, coupled, and nonlinear dynamics of multitethered satellite system render the theoretical analyses impossible. In order to obtain the effects of separation strategies and deployment rate on the deployment for a multitethered chain-type satellite system, parametric analyses are performed via numerical simulations.

A three-body tethered satellite system is the simplest multitethered satellite system and could be deployed upwards and downwards simultaneously without any concern of collisions. This is a special case of multitethered system but could not represent multitethered chain-type satellite system whereas a four-body tethered system has the problems described as set forth in Section 3 during the deployment phase. Hence, a four-body tethered system is regarded as the representative of the multitethered chain-type system and is utilized to investigate the effects of separation strategy on the deployment. Additionally, a three-body tethered system is also used in spite of its peculiarity, and this would contribute to the understanding of the effects of the deployment rate on the dynamic behavior of deployment. As a result, both of them are employed to study these effects.

All the satellites are assumed to be identical with a mass of $200 \mathrm{~kg}$, which is helpful to concentrate attention on the issues attractive. The motion of the center of mass of system is treated as unperturbed Keplerian with an eccentricity of 0.025 and semimajor axis as $6628 \mathrm{~km}$. 


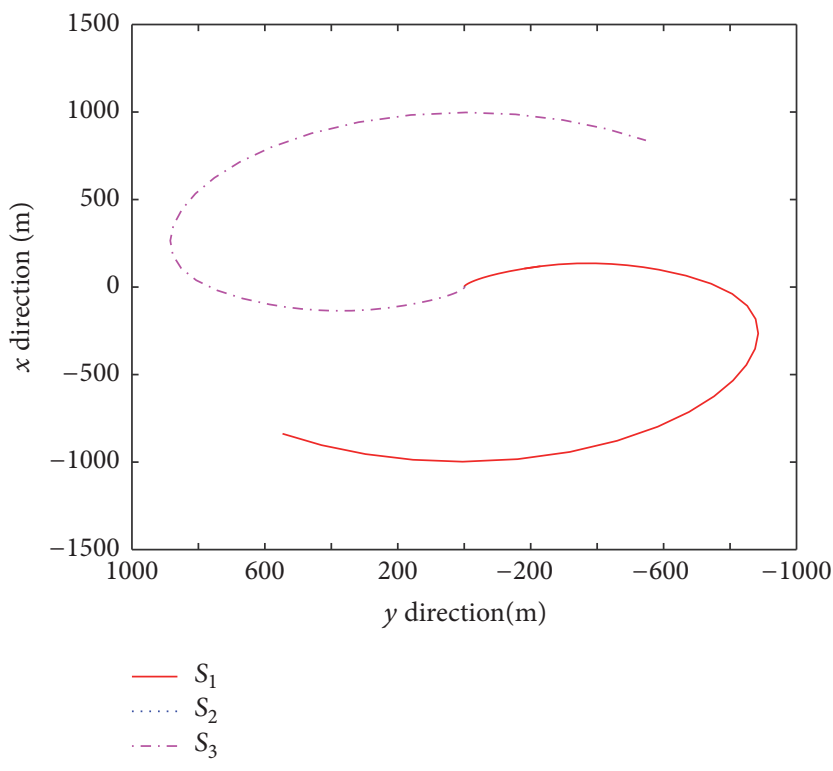

(a)

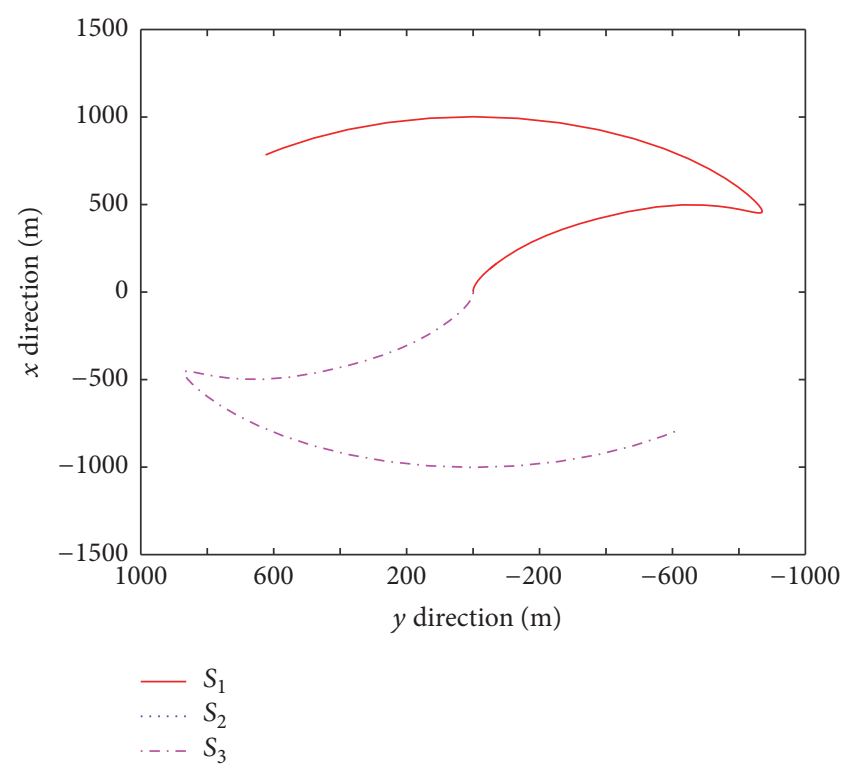

(b)

FIGURE 4: In-plane configurations of system during deployment with different control methods: (a) first method and (b) second method.

5.1. Effect of Deployment Rate on the Dynamic Behavior of System. The nonlinear dynamic behavior of a three-body tethered satellite system is now numerically analyzed for two different deployment rates of the tethers, whose lengths are identical. Assuming the desired lengths of both tethers are $1 \mathrm{~km}$, the relevant parameters of the system are selected as (27) and the deployment with that is called Case A. Computer simulations are carried out and the numerical results except for the position of satellites, which has been shown in Figure 4, are given in Figure 5.

The station-keeping phase after the deployment is also given to validate the efficiency of the selected parameters. Clearly, with the parameters chosen in (27), two tethers are deployed synchronously and the deployment finishes at 2072 s. Then, the system enters into station-keeping phase as shown in Figure 5(a). As depicted in Figure 5(b), the variations of in-plane angle of two tethers coincide during deployment, and the deviation of them is a sinusoidal wave caused by the tiny discrepancy at the end of deployment. The out-of-plane angles maintaining zero during all the procedure could be seen in Figure 5(c), and the configuration during deployment is represented in Figure 5(d). The configuration of system coincides with the distribution of satellites shown in Figure 4(b) and the mid-satellite retains at centroid of the system. Since the out-of-plane angles are zeros all through, the motion besides the orbital plane does not exist and the motion of system could be confined into orbital plane, which can simplify the dynamic equations and help analyze the deployment theoretically. Thus, the equation of in-plane angle in (16) is rewritten as

$$
\begin{aligned}
\ddot{\theta}_{k}= & -3 \mu R^{-3} \sin \theta_{k} \cos \theta_{k}-2 \frac{\dot{l}_{k}}{l_{k}}\left(\dot{\theta}_{k}+\omega\right)-\dot{\omega} \\
& +\frac{\mathbf{T}_{k+1}}{m_{k+1}} \sin \left(\theta_{k+1}-\theta_{k}\right)-\frac{\mathbf{T}_{k-1}}{m_{k}} \sin \left(\theta_{k}-\theta_{k-1}\right) .
\end{aligned}
$$

Through (28), it could be concluded that the in-plane angle of $k$ th tether is affected by its length, deployment rate, in-plane angles, tether tensions, and initial value. After setting the orbit of the system and initial separation parameters, the deployment rate plays a key role in the in-plane motion of system. To confirm that, another numerical simulation is carried out with the identical initial parameters of the system while the parameters related to deployment rate are as follows:

$$
\begin{aligned}
L_{21} & =752 \mathrm{~m}, \\
L_{21} & =751 \mathrm{~m}, \\
c_{1} & =4 \times 10^{-3} \mathrm{~s}^{-1}, \\
s_{1} & =50 \mathrm{~m}, \\
c_{2} & =8 \times 10^{-3} \mathrm{~s}^{-1}, \\
s_{2} & =25 \mathrm{~m} .
\end{aligned}
$$

The in-plane motion of deployment with control parameters described in (29) is denoted as Case B. The numerical simulations are shown in Figure 6. It is worthy to note that if the initial out-of-plane angle is zero, then the out-of-plane angle would always be zero. Hence, the figures of out-ofplane are omitted hereafter. In this situation the deployment rates of tethers are not identical any more. Figure 6(a) shows that the second tether finishes its deployment faster than the first tether. Hence the first tether is not aligned with the second tether and the position of $S_{2}$ does not coincide with centroid of system due to the distinct length of two tethers during the deployment, as shown in Figures 6(d) and $6(\mathrm{c})$, respectively. The in-plane motions of tethers are not synchronous either and the deviation of the two in-plane angles experiences a sinusoidal-like variation. Additionally, 


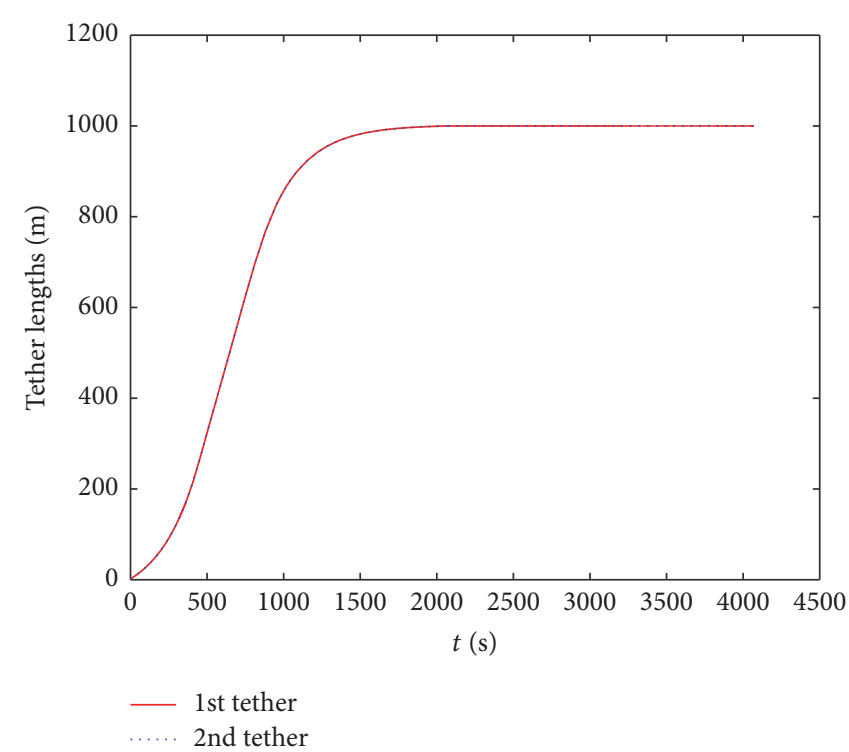

(a)
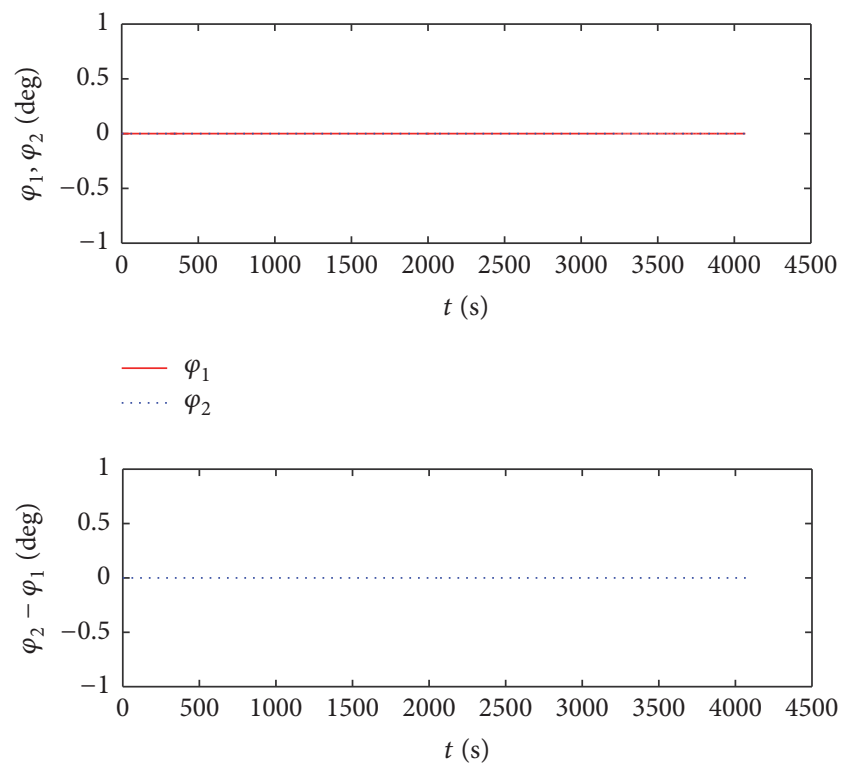

(c)

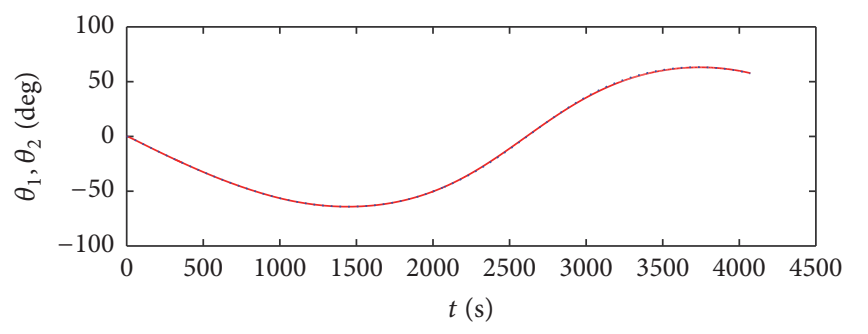

$\theta_{1}$

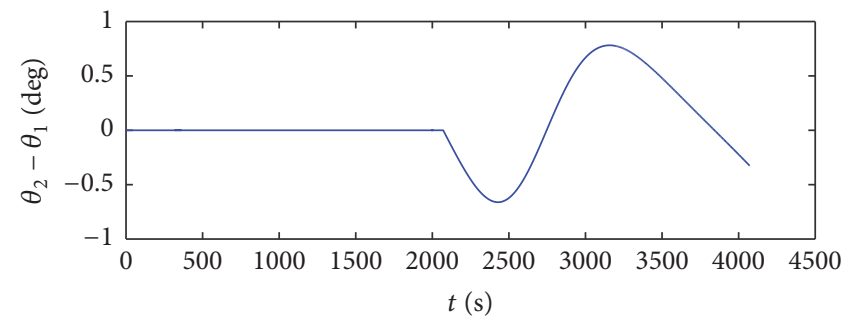

(b)

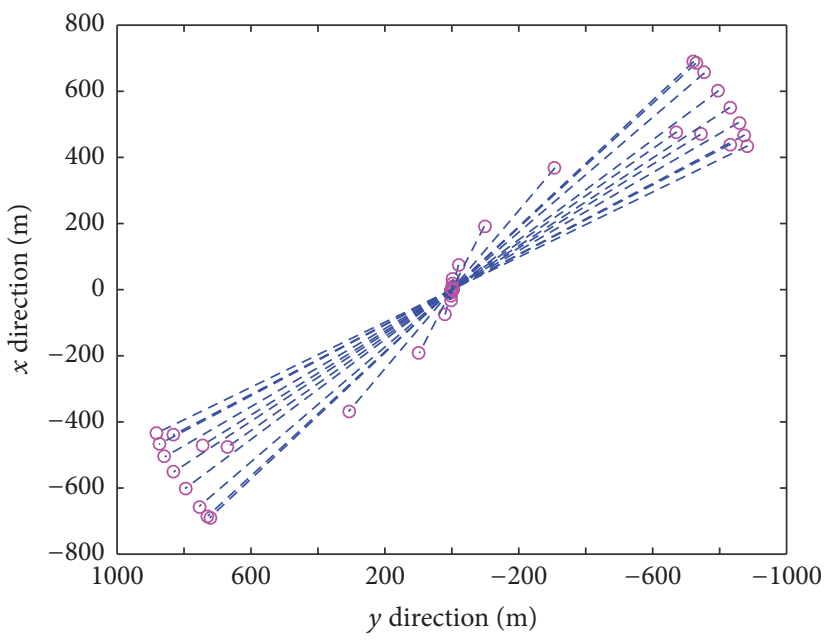

(d)

Figure 5: Dynamic behaviors of Case A: (a) length variation of tethers, (b) in-plane angles and the deviation of them, (c) out-of-plane angles and the deviation of them, and (d) the configuration of system in orbital plane during deployment.

both two angles exceed $90^{\circ}$ during the station-keeping phase, which is undesirable for space missions.

Comparing Figures 5 and 6, we could conclude that for the a three-body tethered satellites system with identical distance between satellites, if the initial states of two tethers for deployment are identical thus the control parameters should be equal as well, such that the in-plane motion of two tethers is synchronous, which is beneficial for the missions. As for the system with two distinct tethers, the related parameters of both tethers should be proportional to desired length of them to obtain a symmetrical in-plane motion of two tethers since the in-plane motion is essentially driven by the deployment rate, as described in (28). For a system with two tethers of $1 \mathrm{~km}$ and $500 \mathrm{~m}$, that is, $L_{1 f}=1 \mathrm{~km}, L_{2 f}=$ $500 \mathrm{~m}$, the parameters should be designed as

$$
\begin{aligned}
& L_{10}=2 \mathrm{~m}, \\
& L_{20}=1 \mathrm{~m}, \\
& L_{11}=250 \mathrm{~m}, \\
& L_{21}=125 \mathrm{~m}, \\
& L_{12}=752 \mathrm{~m},
\end{aligned}
$$




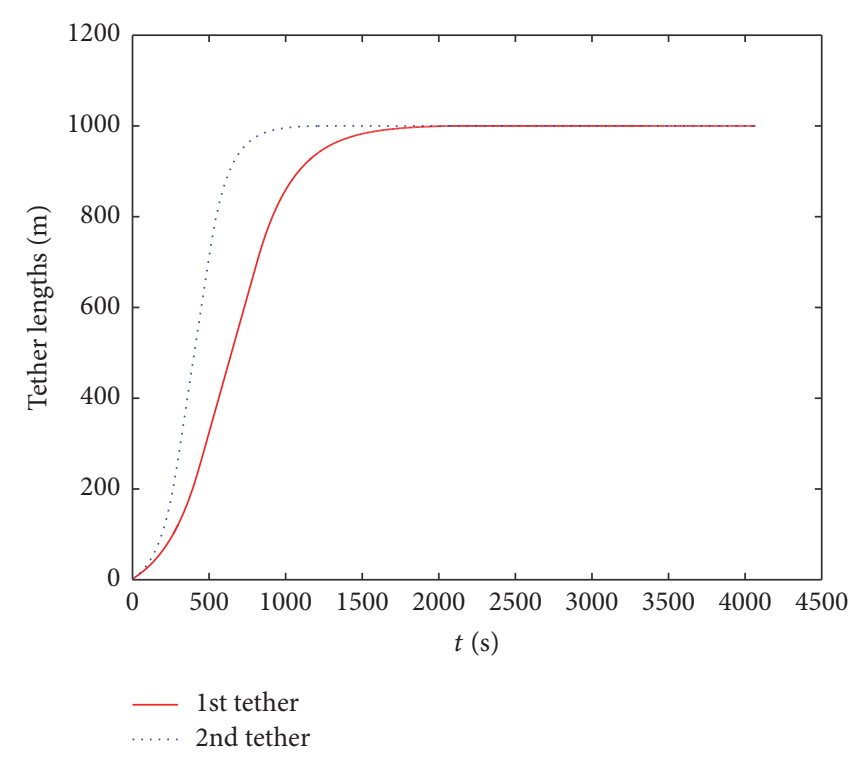

(a)

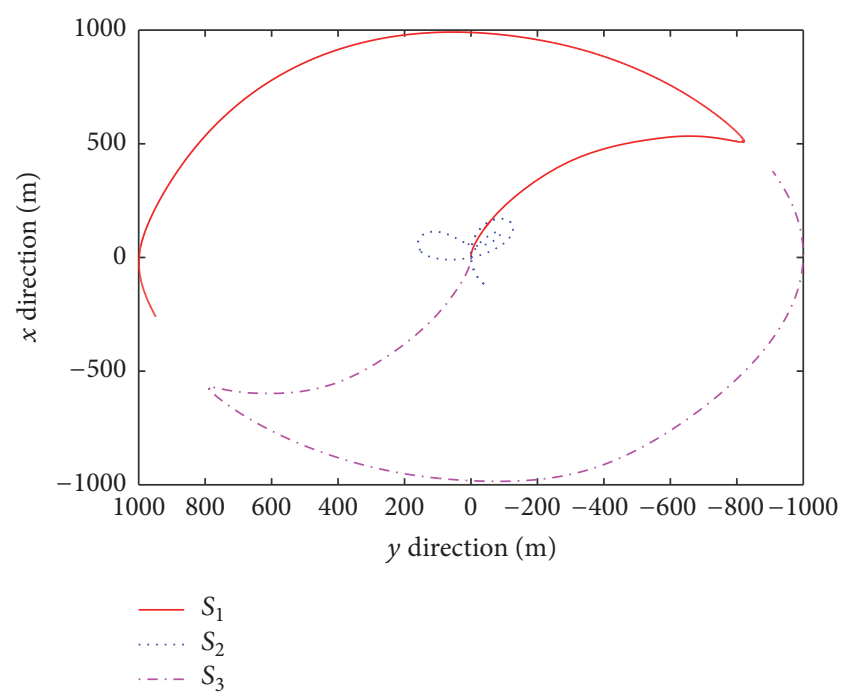

(c)
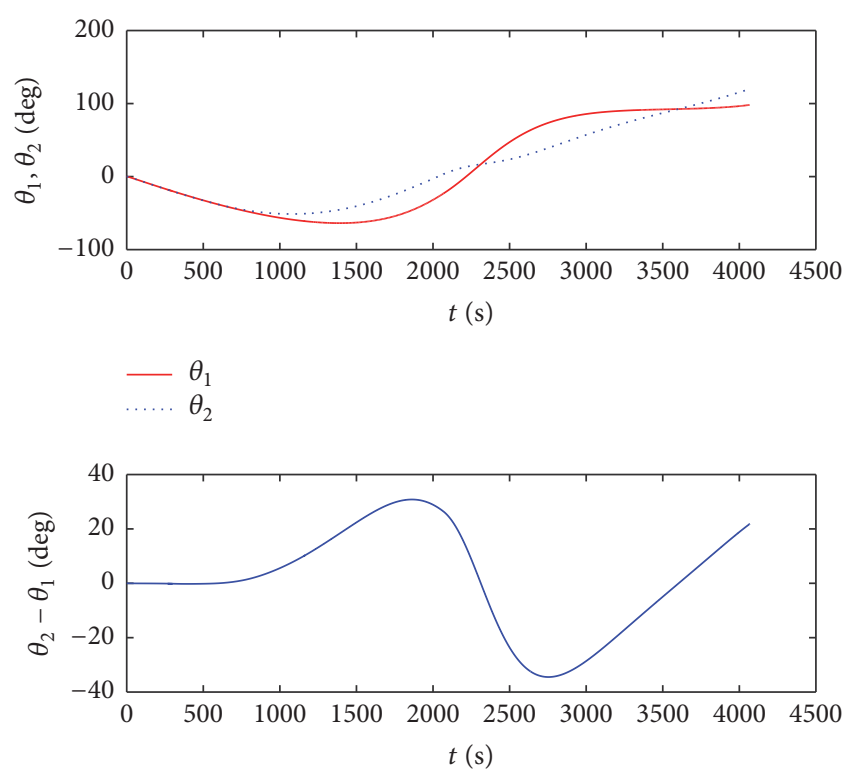

(b)

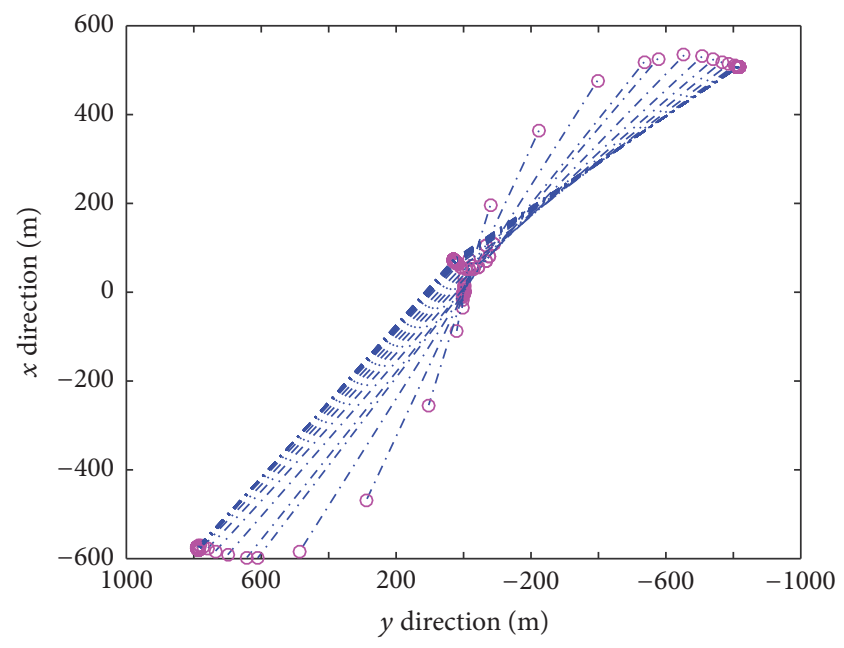

(d)

FIGURE 6: Dynamic behaviors of Case B: (a) length variation of tethers, (b) in-plane angles and the deviation of them, (c) in-plane position of satellites, and (d) the configuration of system in orbital plane during deployment.

$$
\begin{aligned}
L_{22} & =376 \mathrm{~m}, \\
\theta_{10} & =\theta_{20}=0, \\
\dot{\theta}_{10} & =\dot{\theta}_{20}=0, \\
c_{1} & =c_{2}=4 \times 10^{-3} \mathrm{~s}^{-1}, \\
s_{1} & =50 \mathrm{~m}, \\
s_{2} & =25 \mathrm{~m} .
\end{aligned}
$$

The numerical simulation of this case is carried out to validate the analysis and dynamic behavior of in-plane motions are similar with Figure 5 except for the length variation of tethers. For brevity, the figure of simulation results is omitted here.

5.2. Effect of Separation Strategy on the Dynamic Behavior of System. As mentioned before, a four-body tethered satellite system is more representative for multitethered satellite system than a three-body tethered system, as it could not be initialized by one separation. The separation strategy plays an important role in the deployment of a four-body tethered chain-type satellite system. Therefore, the numerical simulations of the deployment for a four-body tethered chain-type satellite system are performed to investigate its effect.

Upon the results of the deployment of the three-body tethered system, a four-body tethered satellite system separated simultaneously is deployed via numerical method. 
Firstly, the system is split into two identical parts via spring mechanism, and each part includes two satellites. The system during this period could be regarded as a two-body tethered system. After the tether connecting these two parts is totally deployed, each part will separate into two satellites along the direction of the tether. There are two approaches of separation, which have been expressed in Section 3. The dynamic behaviors of both separation approaches are numerically performed to investigate their effects on the deployment. According to the process of the deployment, the deploy sequences of tethers could be described as $2 \rightarrow 1,3$. The second separation initializing the deployment of tethers 1 and 3, via spring mechanism, is denoted as Case C, and the other approach by separation bolt is called as Case D. The four-body tethered system includes the three-body tethered system mentioned above and another satellite, whose mass is also $200 \mathrm{~kg}$, joining the three-body tethered system via a $1 \mathrm{~km}$ tether. Moreover, the semimajor axis and eccentricity of the orbit of centroid of system are also $6628 \mathrm{~km}$ and 0.025 . The initial parameters of the system after the first separation and the parameters relevant to control are given as follows:

$$
\begin{aligned}
L_{20} & =2 \mathrm{~m}, \\
\theta_{20} & =\dot{\theta}_{20}=0, \\
\varphi_{20} & =\dot{\varphi}_{20}=0, \\
L_{21} & =250 \mathrm{~m}, \\
L_{22} & =760 \mathrm{~m}, \\
c_{2} & =4 \times 10^{-3} \mathrm{~s}^{-1}, \\
s_{2} & =50 \mathrm{~m} .
\end{aligned}
$$

When the 2nd tether is fully deployed, the second separation is implemented via spring or separation bolt, corresponding to Cases C and D, respectively. The subsequent state of system for the two cases could be determined by (23) and (24) and it is worthy to note that the initial lengths of these two tethers are obtained by assuming that they have deployed for $2 \mathrm{~s}$ with the initial velocity along the separation direction. The numerical simulations of these two cases are shown in Figures 7 and 8.

The dynamic responses of a four-body tethered satellite system separated via spring mechanism twice are depicted in Figure 7. The second separation has an evident effect on the fully deployed 2nd tether, as shown in Figure 7(a). It induces a longitudinal oscillation, which could be seen from the lager image, due to the velocity of satellites $S_{2}$ and $S_{3}$ obtained from the separation. The in-plane motion of system during deployment and after could be viewed from Figures 7(b) and 7(c), which correspond to the inplane angle of tethers and distribution of satellites in orbital plane, respectively. The in-plane angles of 1st tether and 3rd tether vary consistently after the separation. However, inplane angles of all the tethers continually increase, which lead to a rotation around the centroid of the system and the two outside tethers move symmetrically. The configuration of the system during deployment could be seen in Figure $7(\mathrm{~d})$, and the 2nd tether is not plotted after the second separation to display the configuration during the first phase. Clearly, at the end of the deployment, the tethers almost align along the horizontal line, and with the rational speed of tethers at that moment, the system could go across the horizontal line without control, which will raise a rotation of the system.

The dynamic motions responding to the approach of separation bolt are given in Figure 8. Similar to the above results, the 1st and 3rd tether swing synchronously in the orbital plane. Hence the deviations of in-plane angle between them and 2nd tether are opposite, as plotted in Figure 8(b). Unlike the first separation approach, the satellite $S_{2}$ and $S_{3}$ get no extra velocity from the separation. Therefore, comparing to Figure 7(a), the deployment of 1st and 3rd tether take more time since the smaller initial velocity obtained from the separation, which is shown in Figure 8(a). Moreover, the longitudinal oscillation of 2 nd tether is weaker but with a higher frequency determined by the tether material, as shown in the close-up. The configuration of system in Figure 8(d) shows that at the end of the deployment the 1st and 3rd tether already start to swing back to the local vertical, and they will move like a pendulum after the deployment without any control, which can be seen in Figure 8(c).

Comparing these two separation approaches, the system completes deployment faster with the former method but will enter a state of rotation without control, which is undesirable for the space missions. In contrast, the system costs more time using the latter approach and will be in a simple pendulum motion in the state-keeping phase. Though, the system could be controlled to align the vertical line after the deployment no matter which one is chosen. However, the approach of separation bolt is more attractive from the energy consumption point.

As follows, the other separation strategy, that is, splitting successively, is also performed via numerical simulations. Similarly, the system is separated into two parts and $S_{1}$ separates from other satellites to achieve the deployment of the 1st tether. Then when the 1st tether grows to the desired length, $S_{2}$ divorces from the retained satellites by spring mechanism or separation bolt. Subsequently, $S_{3}$ and $S_{4}$ split up as the deployment of the 2 nd tether finishes. The deployments with two separation approaches are denoted as Case E and Case F, respectively. The system parameters are same as before and the equivalent parameters in both cases related to control are as follows:

$$
\begin{aligned}
L_{10} & =2 \mathrm{~m}, \\
\theta_{10} & =\dot{\theta}_{10}=0, \\
\varphi_{10} & =\dot{\varphi}_{10}=0, \\
L_{11} & =L_{21}=L_{31}=250 \mathrm{~m}, \\
L_{12} & =L_{22}=760 \mathrm{~m}, \\
L_{32} & =752 \mathrm{~m}, \\
c_{1} & =c_{2}=c_{3}=4 \times 10^{-3} \mathrm{~s}^{-1}, \\
s_{1} & =50 \mathrm{~m} .
\end{aligned}
$$




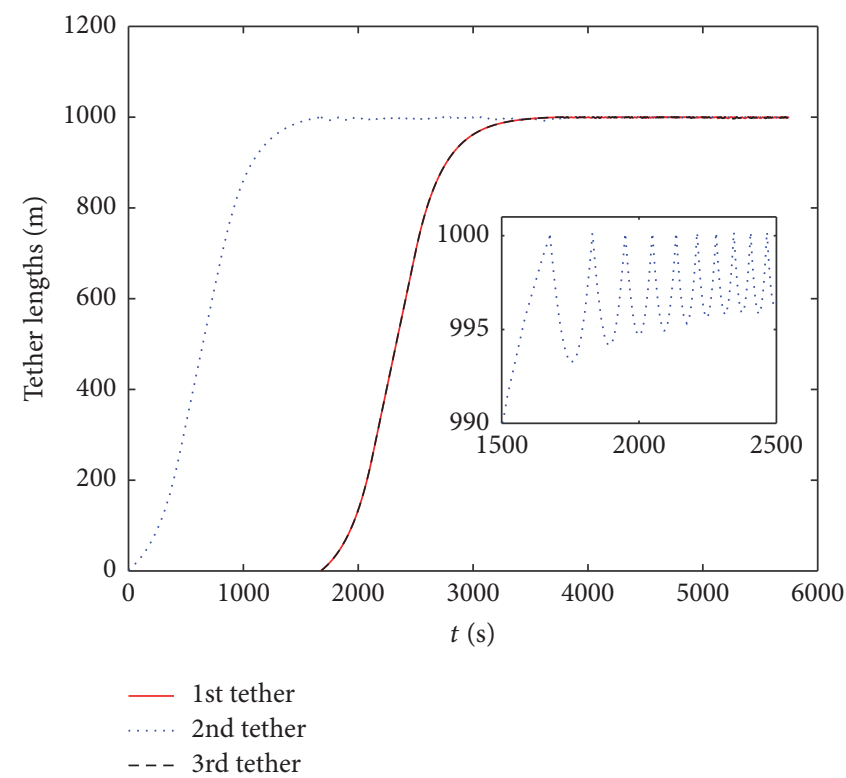

(a)

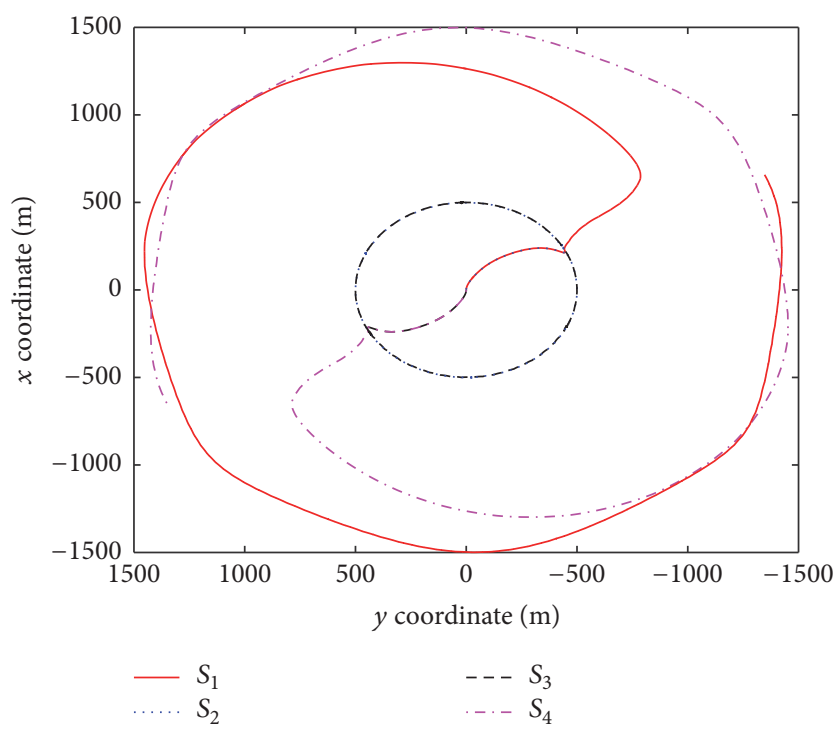

(c)

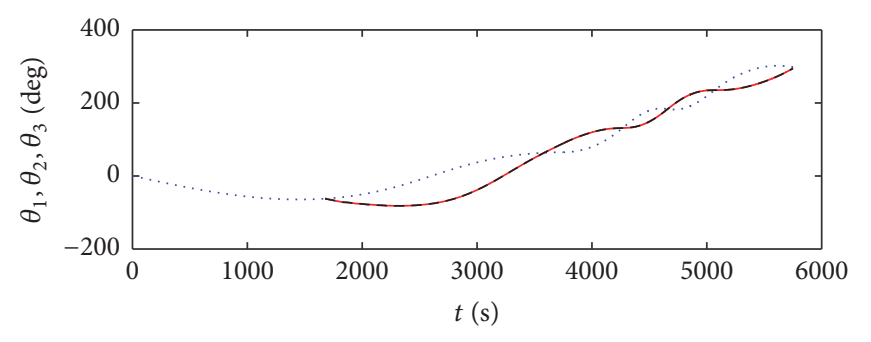

$\begin{array}{cc} & \theta_{1} \\ \ldots . . . & \theta_{2} \\ \ldots-- & \theta_{3}\end{array}$

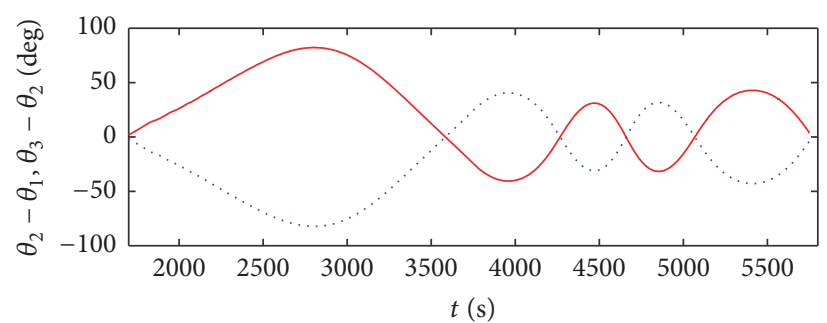

$-\theta_{2}-\theta_{1}$

$\theta_{3}-\theta_{2}$

(b)

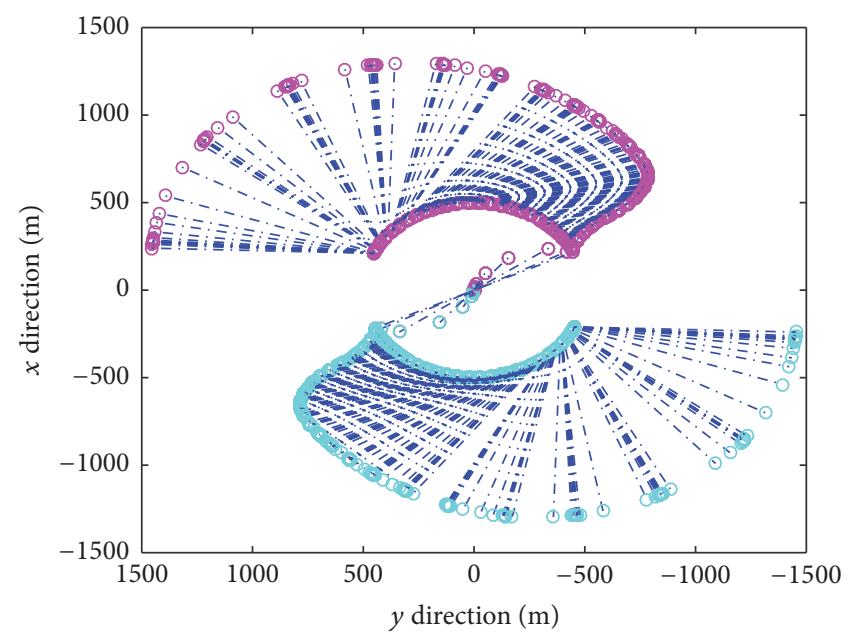

(d)

FIGURE 7: Dynamic behaviors of Case C: (a) length variation of tethers, (b) in-plane angles and the deviations of them, (c) in-plane position of satellites, and (d) the configuration of system in orbital plane during deployment.

The distinct factors of these two cases are

$$
\begin{aligned}
& \text { Case E: } s_{2}=s_{3}=50 \mathrm{~m}, \\
& \text { Case F: } s_{2}=s_{3}=10 \mathrm{~m} .
\end{aligned}
$$

The dynamic behavior corresponding to Case $\mathrm{E}$ is given in Figure 9. The longitudinal oscillation of 1st tether induced by the separation could be seen in Figure 9(a) and there is almost no damping without control. The in-plane motion of system during deployment could be figured out by Figures 9(b), 9(c), and $9(\mathrm{~d})$. It is worthy to note that only the 3rd phase of configuration transformation of system during deployment is depicted and the 2nd tether is denoted by the yellow dot and dash line distinct from other two tethers for clarity. The in-plane angles of tethers continually increase during and after the deployment, and consequently a rotation is raised, which is consistent with Figure 9(c). However, the two outside tethers, 1st and 3rd tether, do not rotate synchronously since the deviations of in-plane angle between them and 2nd tether are not equal during and after the deployment. Hence, the rotation of system is not regular and the orbits of satellites 


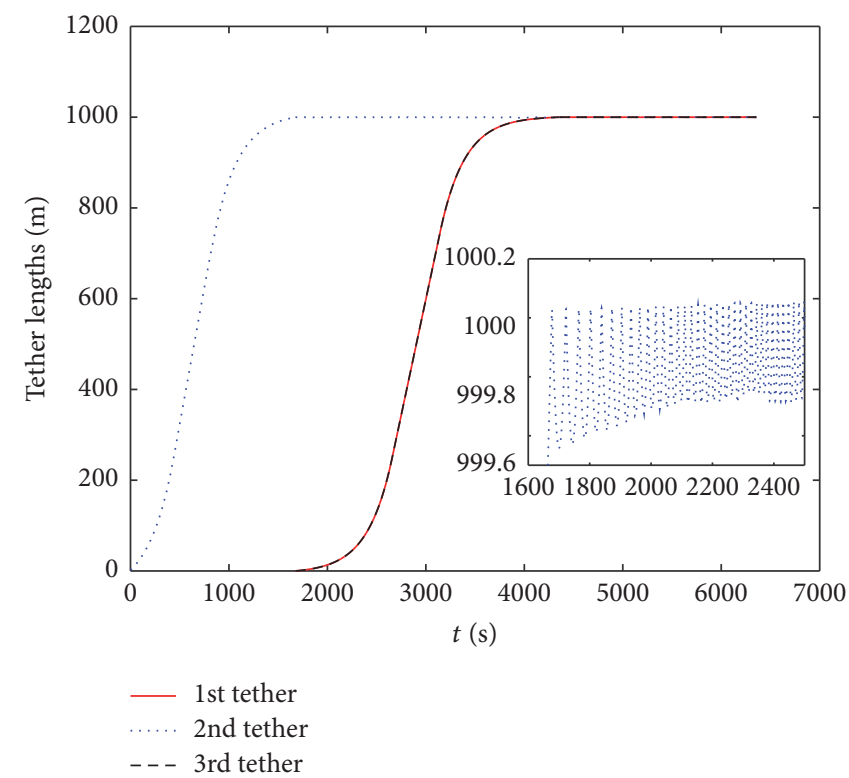

(a)

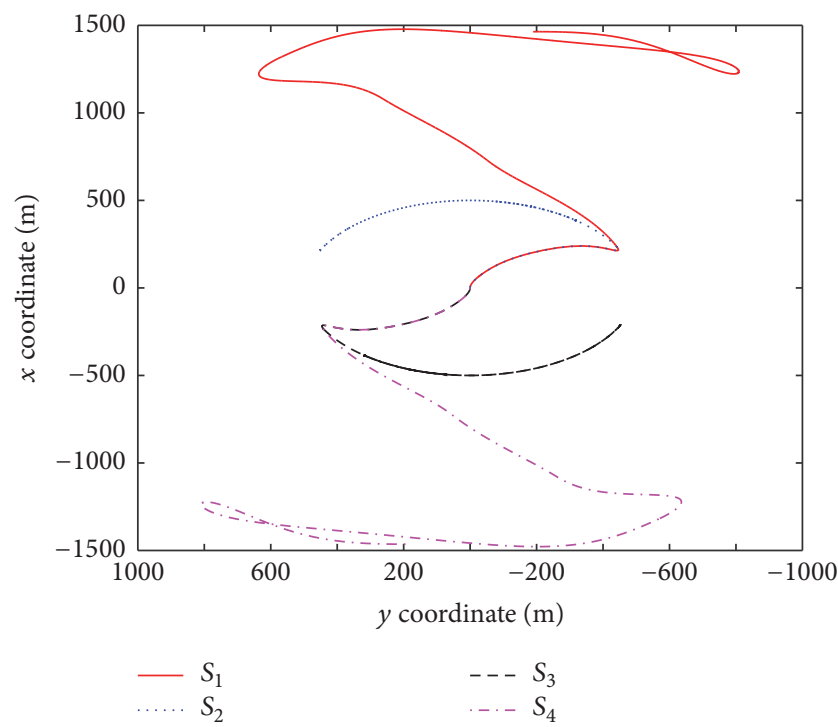

(c)

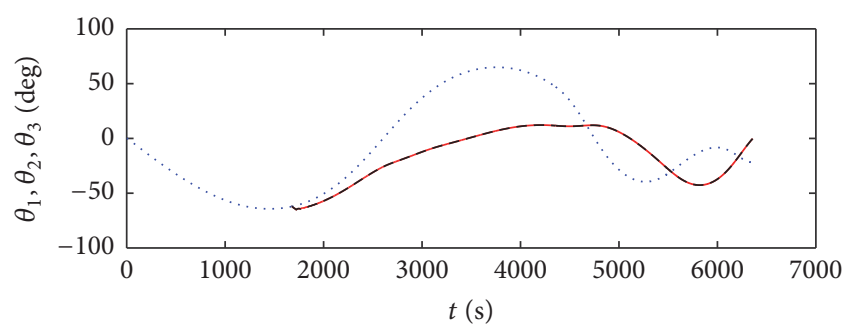

$\begin{array}{ll} & \\ \ldots & \theta_{1} \\ \ldots . & \theta_{2} \\ --- & \theta_{3}\end{array}$

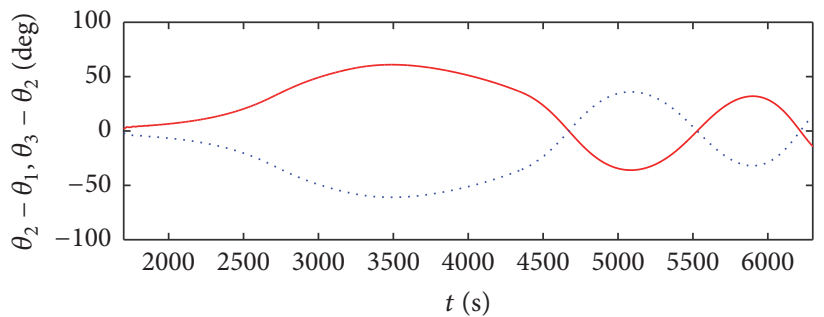

$-\theta_{2}-\theta_{1}$

$\theta_{3}-\theta_{2}$

(b)

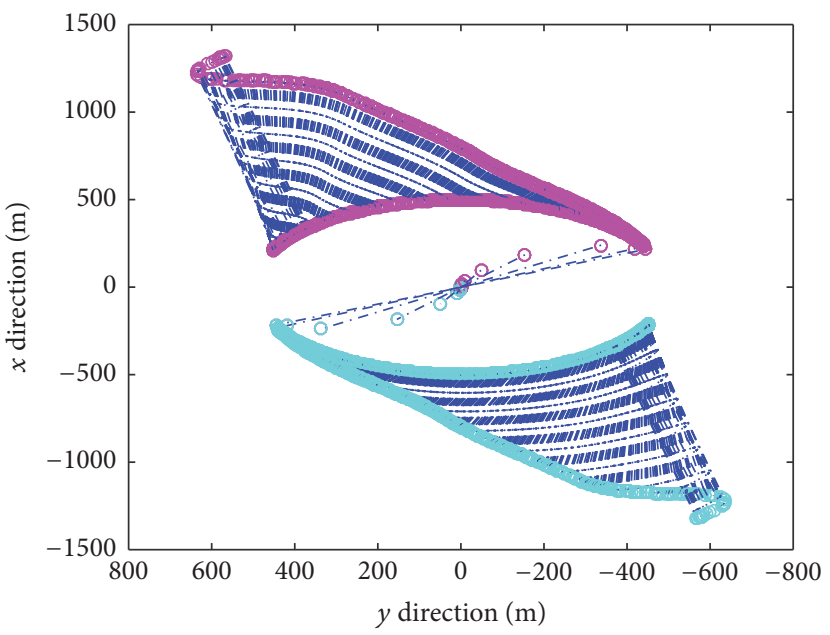

(d)

Figure 8: Dynamic behaviors of Case D: (a) length variation of tethers, (b) in-plane angles and the deviations of them, (c) in-plane position of satellites, and (d) the configuration of system in orbital plane during deployment.

around the center of mass of system are not circular either, which could be seen from Figure 9(c). Moreover, it is clear that the system has already rotated in the 3rd phase of deployment, as shown in Figure 9(d).

The dynamic behavior of Case F is represented in Figure 10. As no extra velocity of satellites linked by the 1st tether obtained from the separation, the longitudinal oscillation of 1st tether is not affected by the separation. Meanwhile, the time consumption of the deployment is longer, which is clearly shown in Figure 10(a). The in-plane angle of deploying tether tends to follow the variation trend of the deployed tether, but the variation ranges of in-plane angle of tethers are not the same, which leads to the distinct deviations between them, as seen in Figure 10(b). However, all of them are less than $90^{\circ}$ which could assure the exclusion of the rotation and the result of Figure 10(c) is consistent with that conclusion. The configuration during the 3rd phase of deployment could be seen as an increasing pendulum, and the tethers swing 


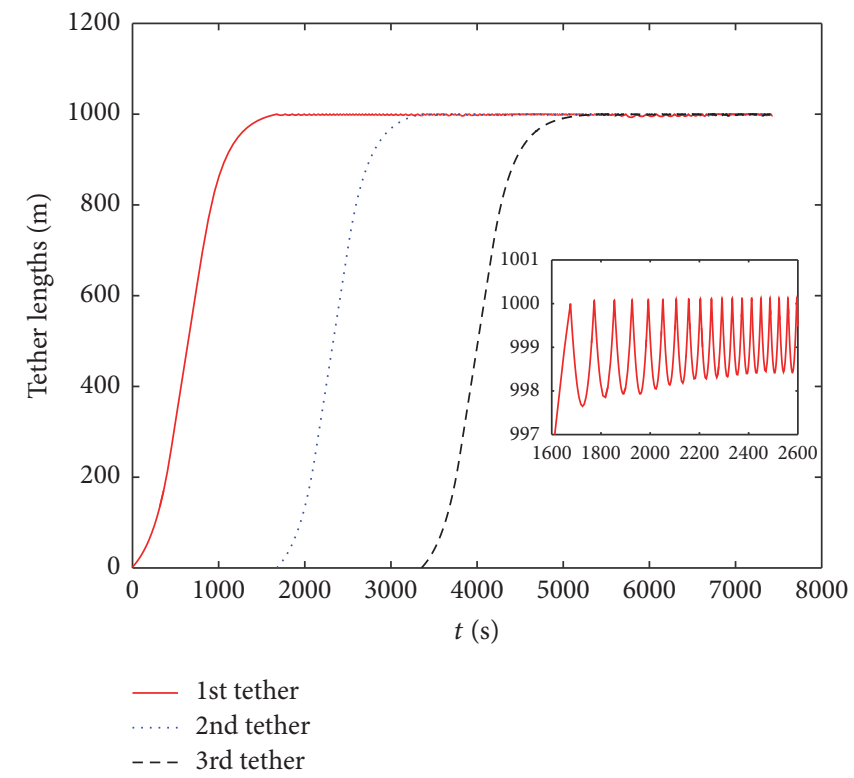

(a)

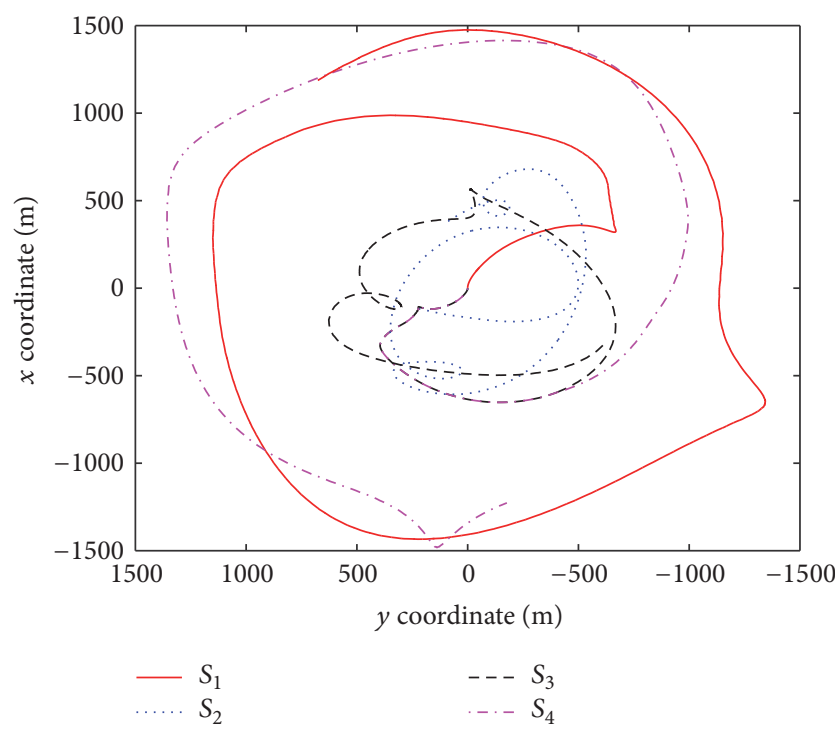

(c)
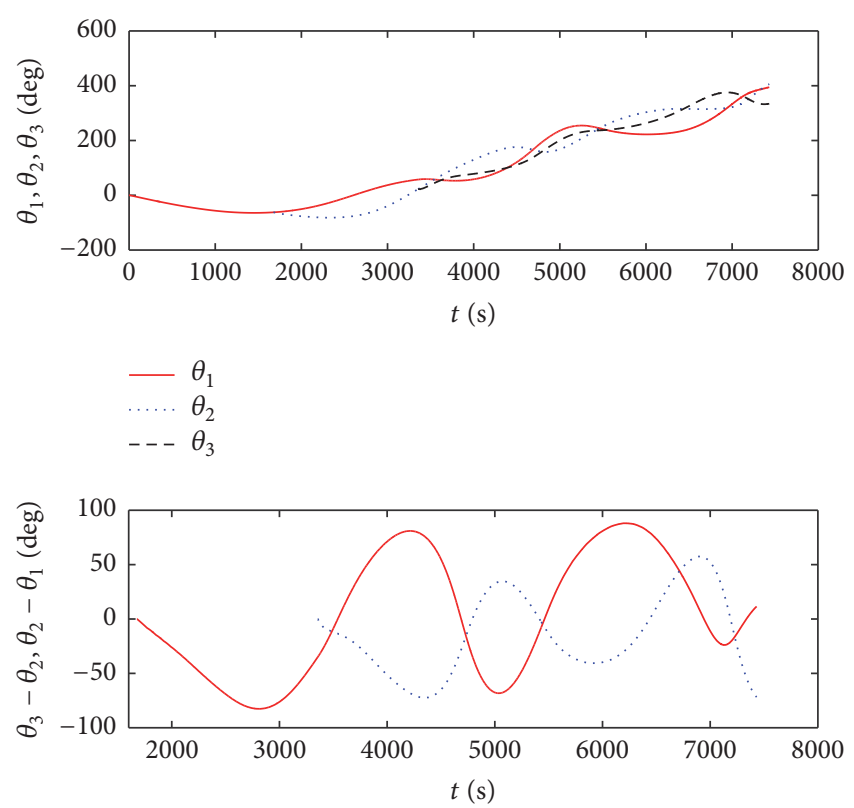

$-\theta_{2}-\theta_{1}$

$\theta_{3}-\theta_{2}$

(b)

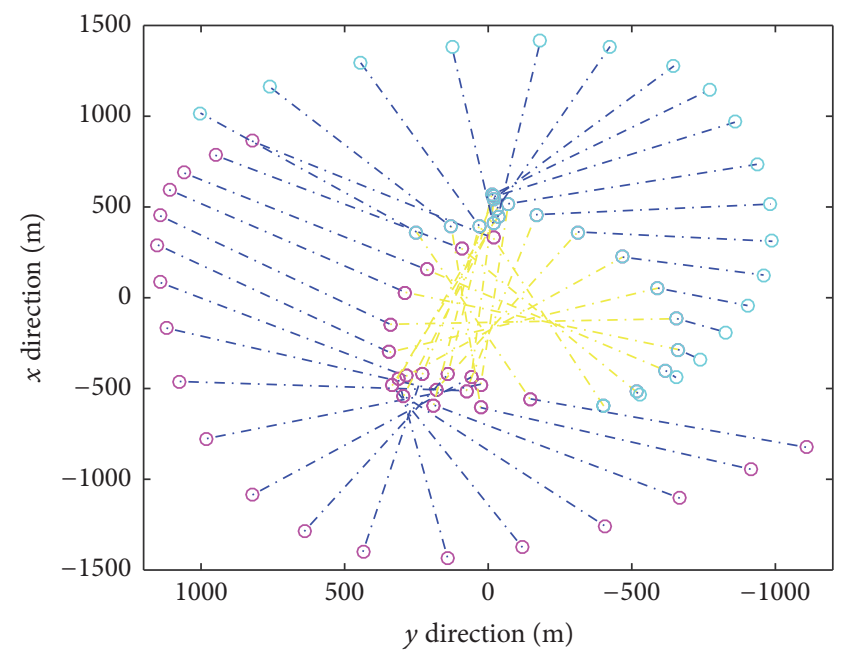

(d)

FIGURE 9: Dynamic behaviors of Case E: (a) length variation of tethers, (b) in-plane angles and the deviations of them, (c) in-plane position of satellites, and (d) the configuration of system in orbital plane during deployment.

back to local vertical in the station-keeping phase. But without damping control, neither the longitudinal oscillation of tethers nor the in-plane motion could be eliminated.

After parametric analyses, the effects of separation strategy on the deployment of multitethered chain-type tethered satellite system could be concluded and the results are as follows: (1) the simultaneous separation method could effectively decrease the time consumption of the deployment, (2) the dynamic behavior of the system separated by virtue of spring mechanism is unprofitable as rotation of system after deployment while the system split by separation bolt ends in a simple pendulum motion, which is better. The latter result could be explained as that the velocity of satellites enlarges by spring mechanism, which will increase the rotational speed of them as well, and the large rotational speed at the end of deployment could make the system into rotation.

\section{Conclusions}

This paper investigates the effects of separation strategy and deployment rate on the dynamic behavior of multitethered 


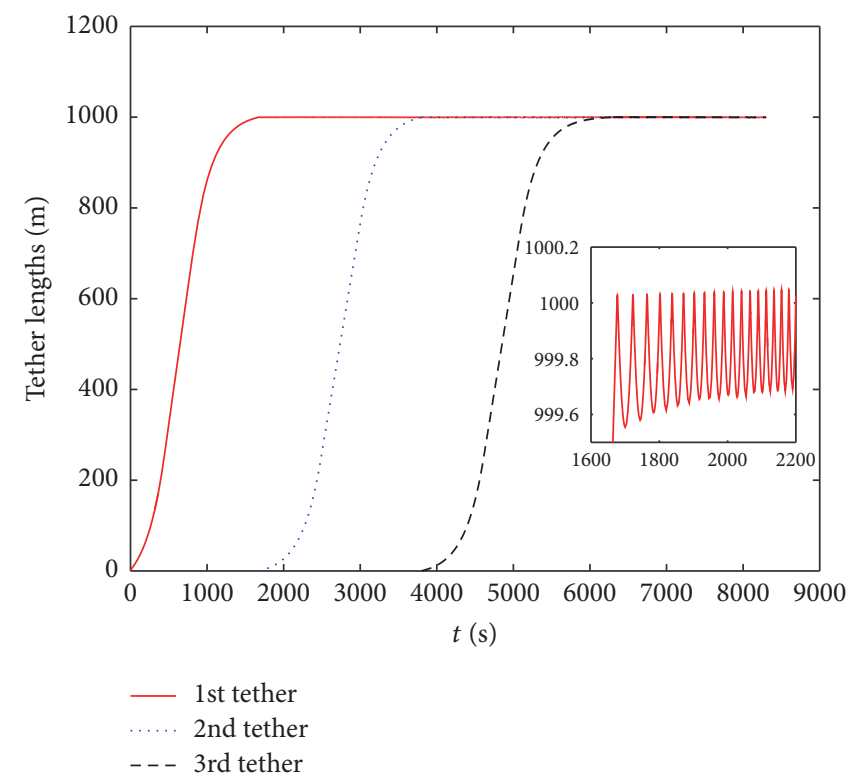

(a)

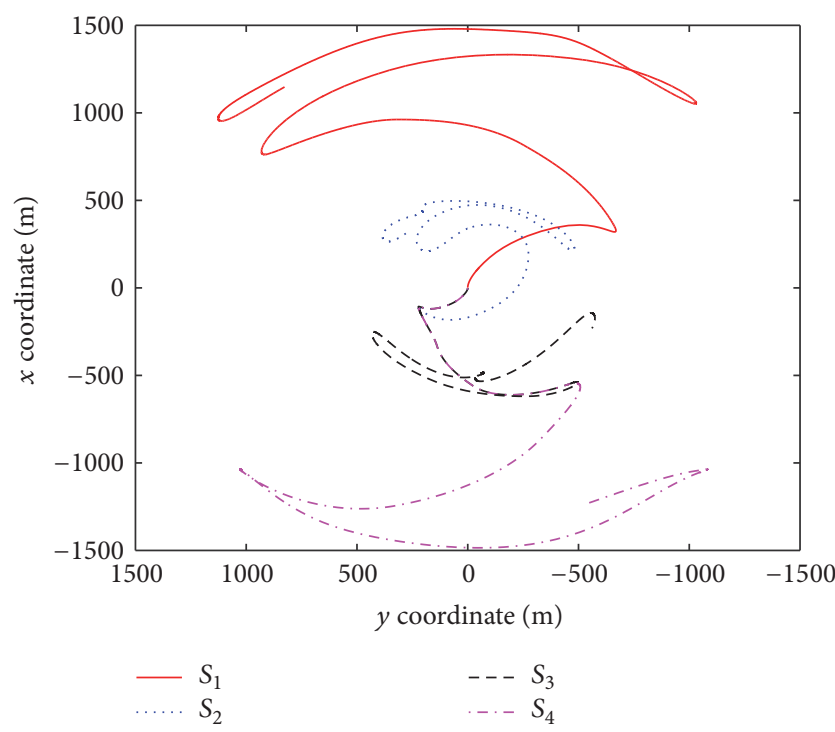

(c)
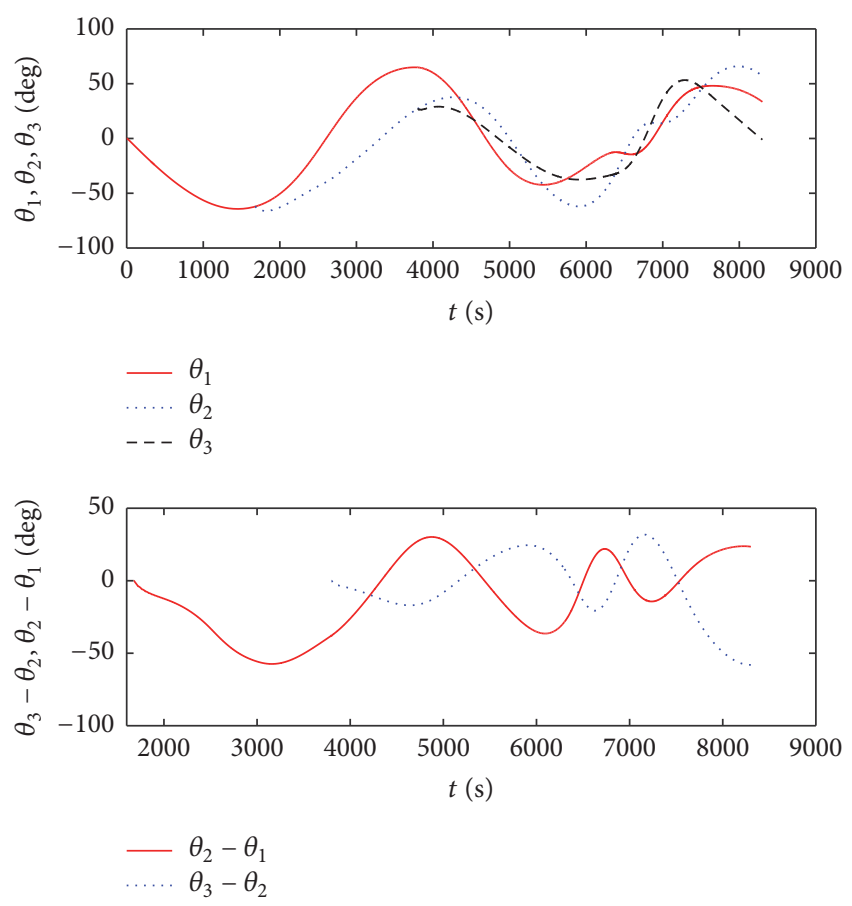

(b)

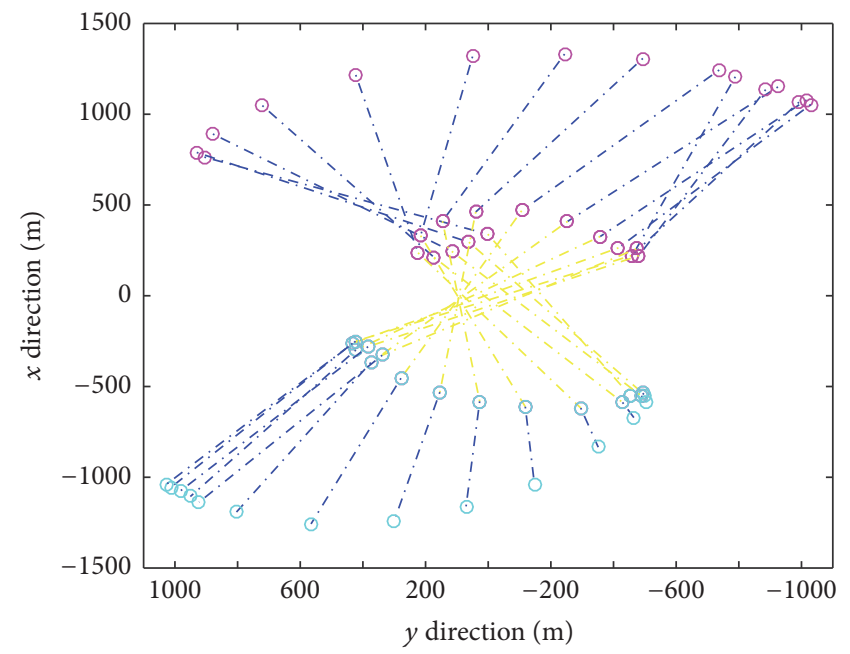

(d)

Figure 10: Dynamic behaviors of Case F: (a) length variation of tethers, (b) in-plane angles and the deviation of them, (c) in-plane position of satellites, and (d) the configuration of system in orbital plane during deployment.

satellite system during its deployment phase. In order to focus on this objective, a simple dynamical model only taking into account the longitudinal and transversal motions of system has been derived using Newtonian Method in absence of external disturbances and tether mass. The multitethered satellite system could be split by spring mechanism or separation bolt and deployed simultaneously or successively using a combination of exponential and uniform velocity deployment. Though a simple dynamical model is applied, the complicated and nonlinear equations still render the analysis difficulty. Therefore, parametric analyses are performed to investigate the effects of separation strategy and deployment rate on the dynamic behavior of deployment for a multitethered chain-type satellite system. A threebody tethered satellite system is deployed simultaneously to obtained the influence of deployment rate while a fourbody tethered satellite system is utilized to study the effect of different separation strategies.

For the simultaneous separation, the time consumption of deployment is less than the other separation strategy, and 
the in-plane angles of the tethers deployed concurrently vary synchronously if the ratio of the deployment rate of them is equivalent to the proportion of their desired length. In contrast, the in-plane motion of tethers which are deployed successively is not consistent even though they have the same desired length and deploy speed. Another result achieved by the numerical simulation is that the four-body tethered satellite system would end in a rotation around the centroid of the system if the satellites are separated by spring mechanism. Hence, by the approach of separation bolt, the system would be in a simple pendulum motion, and it is more preferable. The reason for this phenomena is that the separation carried out by spring mechanism increases not only the deployment rate, but also the rotational speed of system if the separation is not executed along local vertical. Ultimately, the dynamic behavior of multitethered satellite system during deployment is largely affected by the deployment rate and separation strategy.

\section{Competing Interests}

The authors declare that there is no conflict of interests regarding the publication of this paper.

\section{Acknowledgments}

The work is supported by the National Science Foundation of China (no. 91438202).

\section{References}

[1] V. S. Aslanov and A. S. Ledkov, Dynamics of Tethered Satellite Systems, Woodhead, Cambridge, UK, 2012.

[2] H. Troger, A. P. Alpatov, V. V. Beletsky et al., Dynamics of Tethered Space Systems, CRC Press, Boca Raton, Fla, USA, 2010.

[3] Y. Chen, R. Huang, L. He, X. Ren, and B. Zheng, "Dynamical modelling and control of space tethers: a review of space tether research," Nonlinear Dynamics, vol. 77, no. 4, pp. 1077-1099, 2014.

[4] T. R. Kane and D. A. Levinson, "Deployment of a cablesupported payload from an orbiting spacecraft," Journal of Spacecraft and Rockets, vol. 14, no. 7, pp. 409-413, 1977.

[5] A. K. Misra and V. J. Modi, "Deployment and retrieval of shuttle supported tethered satellites," Journal of Guidance, Control, and Dynamics, vol. 5, no. 3, pp. 278-285, 1982.

[6] B. Barkow, A. Steindl, H. Troger, and G. Wiedermann, "Various methods of controlling the deployment of a tethered satellite," Journal of Vibration and Control, vol. 9, no. 1-2, pp. 187-208, 2003.

[7] P. Williams, "Optimal deployment/retrieval of tethered satellites," Journal of Spacecraft and Rockets, vol. 45, no. 2, pp. 324343, 2008.

[8] G. Zhai, J.-R. Zhang, and Z. Yao, "Circular orbit target capture using space tether-net system," Mathematical Problems in Engineering, vol. 2013, Article ID 601482, 11 pages, 2013.

[9] G. Sun and Z. H. Zhu, "Fractional-order tension control law for deployment of space tether system," Journal of Guidance, Control, and Dynamics, vol. 37, no. 6, pp. 2057-2062, 2014.
[10] A. E. Zakrzhevskii, "Method of deployment of a tethered space system along the local vertical," International Applied Mechanics, vol. 51, no. 6, pp. 670-681, 2015.

[11] S. Kalantzis, Dyanmics and Control of Multibody Tethered Systems Using an Order-N Formulation, McGill University, Montreal, Canada, 1994.

[12] A. A. Corrêa and G. Gómez, "Equilibrium configurations of a four-body tethered system," Journal of Guidance, Control, and Dynamics, vol. 29, no. 6, pp. 1430-1435, 2006.

[13] M. Xu, J.-M. Zhu, T. Tan, and S.-J. Xu, "Equilibrium configurations of the tethered three-body formation system and their nonlinear dynamics," Acta Mechanica Sinica, vol. 28, no. 6, pp. $1668-1677,2012$.

[14] G. Avanzini and M. Fedi, "Refined dynamical analysis of multitethered satellite formations," Acta Astronautica, vol. 84, pp. 3648, 2013.

[15] G. Avanzini and M. Fedi, "Effects of eccentricity of the reference orbit on multi-tethered satellite formations," Acta Astronautica, vol. 94, no. 1, pp. 338-350, 2014.

[16] P. Razzaghi and N. Assadian, "Study of the triple-mass tethered satellite system under aerodynamic drag and J2 perturbations," Advances in Space Research, vol. 56, no. 10, pp. 2141-2150, 2015.

[17] D. Alary, K. Andreev, P. Boyko et al., "Dynamics of multitethered pyramidal satellite formation," Acta Astronautica, vol. 117, pp. 222-230, 2015.

[18] W. Jung, A. P. Mazzoleni, and J. Chung, "Nonlinear dynamic analysis of a three-body tethered satellite system with deployment/retrieval," Nonlinear Dynamics, vol. 82, no. 3, pp. 11271144, 2015.

[19] H. Kojima and Y. Fukukawa, "Experimental verification of chaotic librational motion of tethered satellite system in elliptic orbit," in Proceedings of the AIAA Modeling and Simulation Technologies Conference, AIAA 2009-5815, Chicago, Ill, USA, August 2009.

[20] D. Jeyakumar and B. N. Rao, "Dynamics of satellite separation system," Journal of Sound and Vibration, vol. 297, no. 1-2, pp. 444-455, 2006.

[21] F. Zhang, S. Yang, A. Yang et al., "Design of the low impact separation bolt," Initiators and Pyrotechnics, vol. 3, no. 109, p. 14, 2006.

[22] A. K. Misra, Z. Amier, and V. J. Modi, "Attitude dynamics of three-body tethered systems," Acta Astronautica, vol. 17, no. 10, pp. 1059-1068, 1988. 


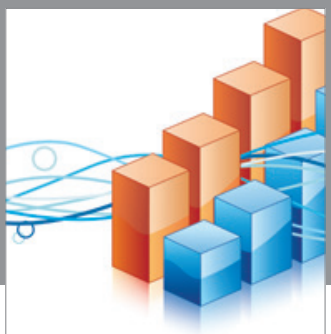

Advances in

Operations Research

vatem alat4

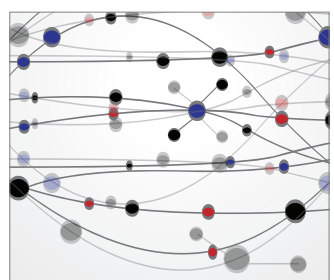

\section{The Scientific} World Journal
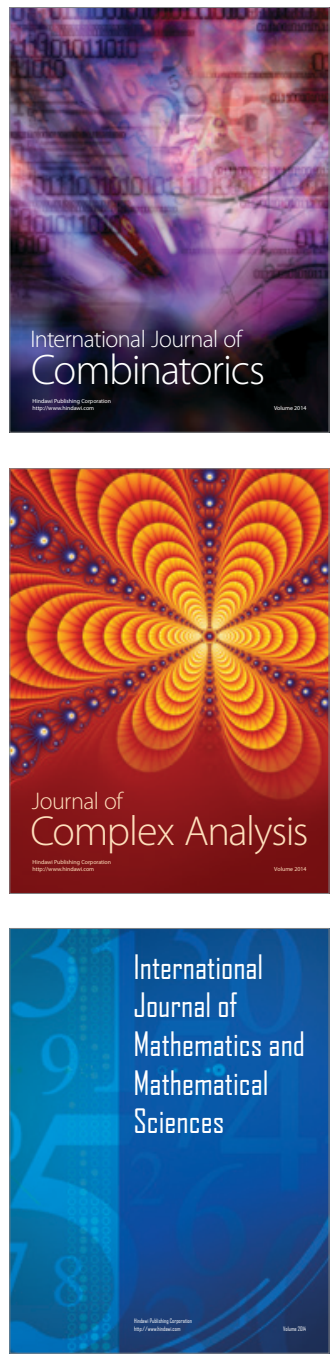
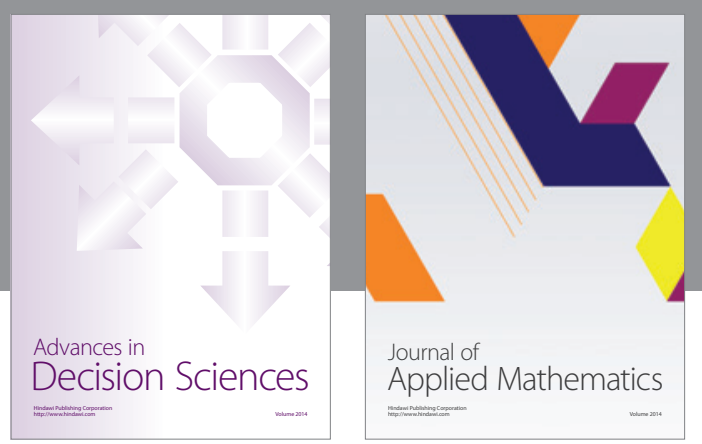

Algebra

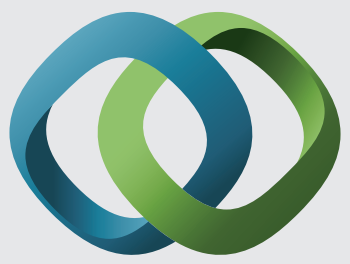

\section{Hindawi}

Submit your manuscripts at

http://www.hindawi.com
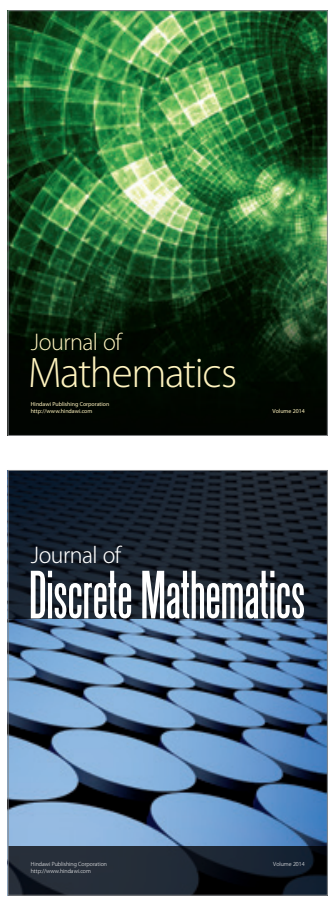

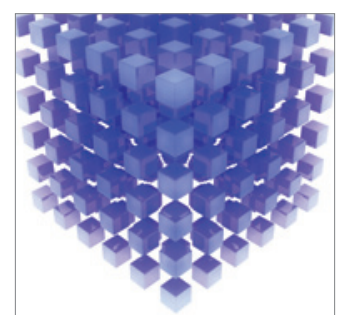

Mathematical Problems in Engineering
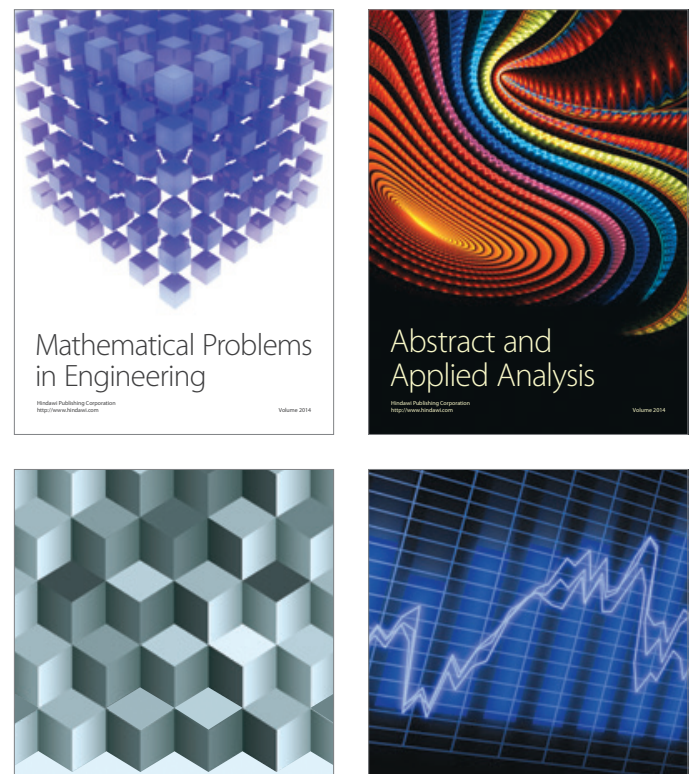

Journal of

Function Spaces

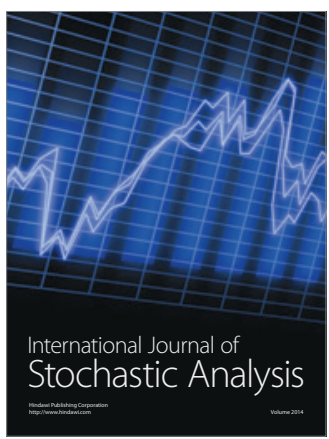

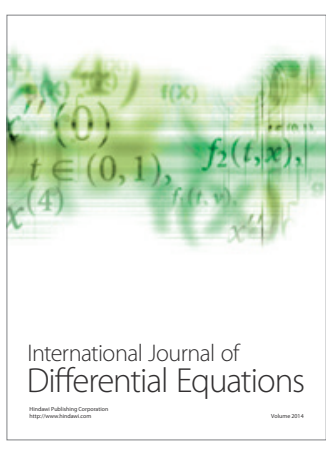
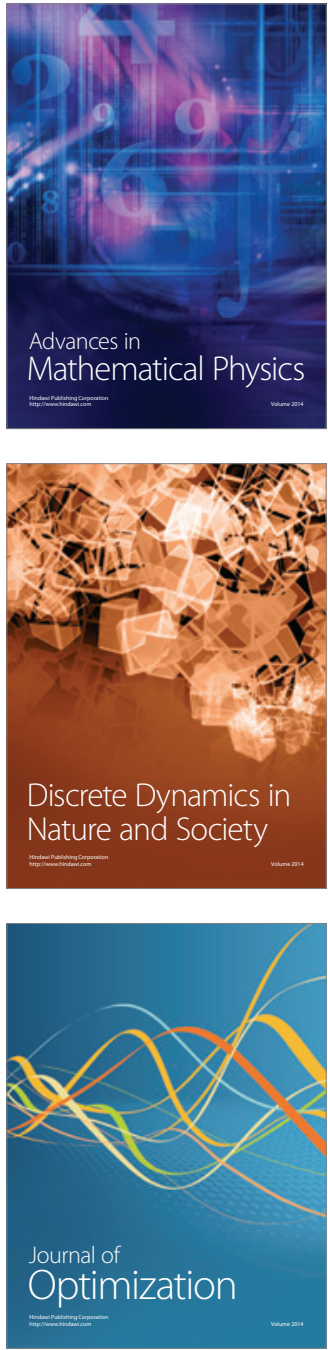